Proceedings of the

International Geometry Center

Vol. 14, no. 1 (2021) pp. 19-48

\title{
On the geometry of $\operatorname{Diff}\left(S^{1}\right)$-pseudodifferential operators based on renormalized traces
}

\author{
Jean-Pierre Magnot
}

\begin{abstract}
In this article, we examine the geometry of a group of Fourierintegral operators, which is the central extension of $\operatorname{Diff}\left(S^{1}\right)$ with a group of classical pseudo-differential operators of any order. Several subgroups are considered, and the corresponding groups with formal pseudodifferential operators are defined. We investigate the relationship of this group with the restricted general linear group $\mathrm{GL}_{\mathrm{res}}$, we define a right-invariant pseudoRiemannian metric on it that extends the Hilbert-Schmidt Riemannian metric by the use of renormalized traces of pseudo-differential operators, and we describe classes of remarkable connections.
\end{abstract}

Анотація. В даній роботі ми вивчаємо геометрію групи інтегральних за Фур'є операторів, яка є центральним розширенням групи $\operatorname{Diff}\left(S^{1}\right)$ за допомогою групи класичних псевдодиференціальних операторів всіх порядків. Розглянуто декілька підгруп та визначено відповідні групи 3 формальними псевдодиференціальними операторами. Ми досліджуємо взаємозв'язок цієї групи з обмеженою загальною лінійною групою $\mathrm{GL}_{\mathrm{res}}$, визначаємо на ній право-інваріантну псевдо-ріманову метрику, яка розширює ріманову метрику Гільберта-Шмідта за допомогою перенормованих слідів псевдо-диференціальних операторів, а також описуємо деякі цікаві класи зв'язностей.

2010 Mathematics Subject Classification: 22E66, 47G30, 58B20, 58J40

Keywords: Fourier-integral operators, infinite dimensional groups, Schwinger cocycle, pseudo-differential operators, renormalized traces, Hilbert-Schmidt metric

Ключові слова: Інтегральні оператори Фур'є, нескінченновимірні групи, коцикл Швінгера, псевдодиференціальні оператори, перенормовані сліди, метрика ГільбертаШмідта

DOI: http://dx.doi.org/10.15673/tmgc.v14i1.1784 


\section{INTRODUCTION}

In the mathematical literature, the introduction of infinite dimensional Lie groups is very often motivated by the use that can be done of such objects which intrinsic properties are difficult to catch. For example, symmetry groups arise in the theory of ordinary differential equations and partial differential equations. Groups of diffeomorphisms arise in the basic theory of differential manifolds, dynamical systems, and have applications in a wide range of examples such as knot theory, stochastic analysis. Manifolds of maps, current groups and gauge groups have their own applications in various models in physics while their intrinsic properties are deeply related to homotopy theory.

The aim of this paper is the description of a family of infinite dimensional Lie groups, derived from a natural interplay between classical pseudodifferential operators and diffeomorphisms, which can be found in the remark first in [33] and in [23], specializing the study when the base manifold is $S^{1}$. Motivations for the introduction of these groups are different in the two works. In [33] the motivation is the full description of a possible structure group for infinite dimensional principal bundles where a Chern-Weil theory can be stated. In [23], the motivation comes from the need of description of the structure group of the space of non-parametrized space of embeddings of a smooth compact boundaryless manifold $M$ to a smooth finite dimensional Riemanian manifold manifold $N$. In both descriptions, the group under consideration is the central extension of a group of diffeomorphisms by a group of classical pseudo-differential operators.

More precisely, the group under consideration in a central extension of the group of diffeomoprhisms $\operatorname{Diff}\left(S^{1}\right)$ by the group $C l^{*}\left(S^{1}, V\right)$ of invertible elements of the algebra $C l\left(S^{1}, V\right)$ of non-formal, classical, maybe unbounded pseudo-differential operators acting on a trivial $n$-dimensional Hermitian bundle $S^{1} \times V$ over $S^{1}$. In such an object, one can derive many infinite dimensional groups:

(1) the loop group $C^{\infty}\left(S^{1}, S U_{n}\right)$

(2) the group of orientation preserving diffeomorphisms $\operatorname{Diff}_{+}\left(S^{1}\right)$

(3) the group generated by $L^{2}$-orthogonal symmetries related to $L^{2}$ orthogonal projections on a finite dimensional vector subspace of $C^{\infty}\left(S^{1}, V\right)$

But beside these examples of groups of bounded operators, which are all subgroups of the restricted unitary group $U_{\text {res }}$ described in [34], we also wish to recover here some non-formal versions of the spaces of elliptic injective classical pseudo differential operators, and in particular the square root of the Laplacian, in order to state results in a framework as general as possible. 
Due to the presence of unbounded pseudo-differential operators, and in particular differential operators of order 1, the Lie algebra of this group cannot be embedded in a group of bounded operators acting on the space of sections $C^{\infty}\left(S^{1}, V\right)$, but only represented in it. As a technical remark, we have to say that we have here an example of non-regular infinite dimensional Lie group, as given in Remark 2.31 adapting a remark from [27]. But another technical feature is that this group does not seem to carry atlas, for the same reasons of presence of unbounded operators, as first described in [2] in the context of formal pseudo-differential operators. But one can anyway consider this group as an "infinite dimensional group" in the way of [12], or as a so-called Frölicher Lie group, see e.g. [27] and references therein, in order to make safe the notion of smoothness. In the section dedicated to the preliminaries, we describe this groups and some of its remarkable subgroups, we recall classical properties of the (zeta-)renormalized traces of pseudo-differential operators, describe useful splittings of the algebra of formal pseudo-differential operators from existing literature, and fully describe the space of formal $\operatorname{Diff}\left(S^{1}\right)$-pseudo-differential operators. This last description is, to our knowledge, not given before this work.

In Section 3, we compare this group with the restricted general linear group and develop the index 2 -form $\lambda$ on it. Indeed, by considering only $S^{1}$ as a base manifold, this enables us to describe more deeply the algebraic and geometric structures derived from the Dirac operator $D$, or more precisely its sign $\epsilon(D)$, which defines a polarization on $L^{2}\left(S^{1}, \mathbb{C}^{k}\right)$, splitting this space into eigenspaces of positive and non-positive eigenvalues. This polarization is described in e.g. [34], is shown here to generalize to unbounded operators in an elegant way, generalizing the remarks initiated in $[18,21]$ on one hand and some geometric constructions using the Lie group of bounded operators

$$
\begin{aligned}
\operatorname{GL}_{\text {res }}\left(S^{1}, \mathbb{C}^{k}\right)=\{u \in \mathrm{GL}( & \left.L^{2}\left(S^{1}, \mathbb{C}^{k}\right)\right) \\
& \text { such that }[\epsilon(D), u] \text { is Hilbert-Schmidt }\}
\end{aligned}
$$

with Lie algebra

$$
\mathcal{L}\left(S^{1}, \mathbb{C}^{k}\right)=\left\{u \in \mathcal{L}\left(L^{2}\left(S^{1}, \mathbb{C}^{k}\right)\right) \text { such that }[\epsilon(D), u] \text { is Hilbert-Schmidt }\right\} .
$$

on the other hand. This Lie group has a central extension, and the corresponding central extension of its Lie algebra is given by the Schwinger cocycle, first found by J. Schwinger in [36]. This 2-cocycle was known as a cocycle on Lie algebras of bounded operators (see e.g. [5,28]). In [21,22] we proved that the Schwinger cocycle can be extended naturally to the algebra $P D O\left(S^{1}, \mathbb{C}^{k}\right)$ of (maybe non classical, maybe unbouded) pseudodifferential operators. Moreover, we gave its relations with a pull-back $c_{+}^{D}$ 
of the Khesin-Kravchenko-Radul cocycle on formal symbols [15, 35], using an appropriate linear extension of the usual trace of trace-class operators. $c_{+}^{D}$ and $\frac{1}{2} c_{S}^{D}$ have the same cohomology class. On loop groups, this (Lie algebra)-cocycle pulls back to the central extension of the loop algebra $[5,18,34]$, while it enables to recover the Gelfand-Fuchs cocycle on $\operatorname{Vect}\left(S^{1}\right),[21]$. We extend here the Lie-algebraic considerations of [21] to the grôu under consideration.

In Section 4 we construct the extension of the Hlibert-Schmidt Hermitian product

$$
(a, b) \mapsto \operatorname{tr}\left(a b^{*}\right)
$$

to the Lie algebra $C l\left(S^{1}, V\right) \rtimes \operatorname{Vect}\left(S^{1}\right)$ by replacing the usual trace of trace-class operators by one of its linear extensions, the zeta-renormalized trace $\operatorname{tr}^{\Delta}$ described in the preliminaries. The first difficulty comes from the fact that $\operatorname{tr}^{\Delta}$ is not tracial, i.e.

$$
\exists(a, b) \in C l\left(S^{1}, V\right): \quad \operatorname{tr}^{\Delta}([a, b]) \neq 0 .
$$

Surprisingly, we obtain a non-degenerate sesquilinear form $(\cdot, \cdot)_{\Delta}$ on $C l\left(S^{1}, V\right)$, which gives rise to a non-degenerate bilinear form $\mathfrak{R e}(\cdot, \cdot)_{\Delta}$ on $C l\left(S^{1}, V\right) \rtimes \operatorname{Vect}\left(S^{1}\right)$. We also show that these forms are not positive, and multiplication operators are isotropic vectors. These two forms then generate, by right-invariant action, pseudo-Hermitian and pseudo-Riemannian metrics on $C l^{*}\left(S^{1}, V\right)$ and $F C l_{\operatorname{Diff}\left(S^{1}\right)}^{*}\left(S^{1}, V\right)$ respectively.

Due to the presence of a pseudo-Hermitian metric, one can ask whether there exists pseudo-Hermitian connections. This is the aim of Section 5, where families of pseudo-Hermitian connections are described. However, there exist some technical difficulties to pass in order to describe the whole family of pseudo-Hermitian connections for $(\cdot, \cdot)_{\Delta}$, due in particular to the actual lack of knowledge on the linear maps acting on $C l\left(S^{1}, V\right)$. We restrict our investigations to connection 1-forms that read as composition by a smoothing operator. Our results are mostly based on the remark that $[a, \epsilon(D)]$ and sas $^{*}$, when $s$ is smoothing, are smoothing operators.

We finish this work by giving some specialization remarks. We describe how $\operatorname{Diff}\left(S^{1}\right)$ acts on the polarization, we give another interpretation of the Schwinger cocycle in terms of the curvature of one of our smoothing connections defined in Section 5, we show that a Levi-Civita type exists for $\mathfrak{R e}(\cdot, \cdot)_{\Delta}$ on the group $F C l_{e e, \operatorname{Diff}\left(S^{1}\right)}^{*}\left(S^{1}, V\right)$ and that it fits with classical formulas for the Levi-Civita connections in finite dimensional settings, and we finish with a blockwise decomposition of $\mathfrak{R e}(\cdot, \cdot)_{\Delta}$ on the group of bounded, even-even class operators $F C l_{e e, \operatorname{Diff}\left(S^{1}\right)}^{0, *}\left(S^{1}, V\right)$. 
Acknowledgements. The author is happy to dedicate this work to Sylvie Paycha on her 60th birthday, 18 years after working under her supervision, with gratitude for this first step into research.

\section{Preliminaries on operators on $S^{1}$}

In this section, we make a moderate use of the notion of diffeology, notion which is not among the main goals of this paper. A classical presentation on diffeologies can be found in [10], and the necessary material is already reviewed in many publications of the author. We refer to [26] and references therein for an updated review of the necessary notions to understand indepth technical properties of the present paper, and to [23] for a preliminary work about the same objects. When precise notions and properties will be necessary, precise citations will be given. For a superficial reading, the reader can replace diffeologies by a «natural differentiation» on operators.

2.1. Basics on pseudo-differential operators. An exposition of basic facts on pseudo-differential operators can be found in [8]. In this section, we only review the definitions and tools that are necessary for this note.

We set $S^{1}=\{z \in \mathbb{C}|| z \mid=1\}$. We shall use for convenience the smooth atlas $\mathcal{A}$ of $S^{1}$ defined as follows:

$$
\begin{aligned}
\mathcal{A} & =\left\{\varphi_{0}, \varphi_{1}\right\} ; \\
\varphi_{n} & : x \in(0 ; 2 \pi) \mapsto e^{i(x+n \pi)} \subset S^{1} \text { for } n \in\{0 ; 1\} .
\end{aligned}
$$

Associated to this atlas, we fix a smooth partition of the unit $\left\{s_{0} ; s_{1}\right\}$. We identify each of these functions with its associated multiplication operator when necessary. An operator $A: C^{\infty}\left(S^{1}, \mathbb{C}\right) \rightarrow C^{\infty}\left(S^{1}, \mathbb{C}\right)$ can be described in terms of four operators

$$
A_{m, n}: f \mapsto s_{m} \circ A \circ s_{n} \text { for }(m, n) \in\{0,1\}^{2} .
$$

A scalar pseudo-differential operator of order $o$ is an operator

$$
A: C^{\infty}\left(S^{1}, \mathbb{C}\right) \rightarrow C^{\infty}\left(S^{1}, \mathbb{C}\right)
$$

such that for all $(m, n) \in\{0,1\}^{2}$,

$$
A_{m, n}(f)=\int_{(0 ; 2 \pi)} e^{-i x \xi} \sigma_{m, n}(x, \xi) \widehat{\left(s_{n} \cdot f\right)}(\xi) d \xi
$$

where $\sigma_{m, n} \in C^{\infty}((0 ; 2 \pi) \times \mathbb{R}, \mathbb{C})$ satisfies the following inequality:

$$
\forall(\alpha, \beta) \in \mathbb{N}^{2}, \quad\left|D_{x}^{\alpha} D_{\xi}^{\beta} \sigma_{m, n}(x, \xi)\right| \leqslant C_{\alpha, \beta}(1+|\xi|)^{o-\beta} .
$$

(In these formulas, the maps $f, s_{n}$ and $A_{m, n}(f)$ are read on the local charts $\varphi_{0}, \varphi_{1}$, but we preferred to only mention this aspect and not to give 
heavier formulas and notations, since the setting for $S^{1}$-pseudo-differential operators is rather more simple than for manifolds of higher dimension).

Let $E=S^{1} \times \mathbb{C}^{k}$ be a trivial smooth vector bundle over $S^{1}$. Let also $s \in \mathbb{R}$. An operator acting on $C^{\infty}\left(S^{1}, \mathbb{C}^{n}\right)$ is a pseudo-differential operator if it can be viewed as a $(n \times n)$-matrix of scalar pseudo-differential operators. A pseudo-differential operator $A$ of order $o$ extends to a linear bounded operator on Sobolev spaces $H^{s}\left(S^{1}, \mathbb{C}^{n}\right) \rightarrow H^{s-o}\left(S^{1}, \mathbb{C}^{n}\right)$. In particular, an order 0 pseudo-differential operator is a bounded operator on $H^{s}\left(S^{1}, \mathbb{C}^{n}\right)$.

A pseudo-differential operator of order $o$ is called classical if and only if its symbols $\sigma_{m, n}$ have an asymptotic expansion

$$
\sigma_{m, n}(x, \xi) \sim_{|\xi| \rightarrow+\infty} \sum_{j=-\infty}^{o}\left(\sigma_{m, n}\right)_{j}(x, \xi),
$$

where the maps $\left(\sigma_{m, n}\right)_{j}: S^{1} \times \mathbb{R}^{*} \rightarrow \mathbb{C}$, called partial symbols, are $j$ positively homogeneous, i.e. for all $t>0$ and $(x, \xi) \in S^{1} \times \mathbb{R}^{*}$,

$$
\left(\sigma_{m, n}\right)_{j}(x, t \xi)=t^{j}\left(\sigma_{m, n}\right)_{j}(x, \xi) .
$$

We also define log-polyhomogeneous pseudo-differential operators. A pseudo-differential operator is log-polyhomogeneous if and only if there exists $o^{\prime} \in \mathbb{N}$ such that its symbols $\sigma_{m, n}$ have an asymptotic expansion

$$
\sigma_{m, n}(x, \xi) \sim_{|\xi| \rightarrow+\infty} \sum_{j=0}^{o} \sum_{k=-\infty}^{o^{\prime}}\left(\sigma_{m, n}\right)_{j}(x, \xi)(\log (|\xi|))^{k},
$$

where the maps $\left(\sigma_{m, n}\right)_{j}: S^{1} \times \mathbb{R}^{*} \rightarrow \mathbb{C}$ are classical partial symbols of order $j$. The number $o^{\prime}$ is called the logarithmic order of the pseudodifferential operator. Of course, classical pseudo-differential operators are log-polyhomogeneous pseudo-differential operators of logarithmic order 0 .

The notion of symbol and partial symbol appear local (dependent on the charts of the atlas) in view of these definitions. However, in this very special case of atlas on $S^{1}$, where the changes of coordinates are translations, one can see (with the formulas of change of coordinates given in e.g. [8]) that the partial symbols of a pseudo-differential operator can be defined globally by

$$
\sigma_{j}=\sum_{\left(m, n \in\{0,1\}^{2}\right.}\left(\sigma_{m, n}\right)_{j}
$$

There is another way to define globally the partial symbols of an operator is in [4], see e.g. [39], using linearizations of the manifold. This second way to define the formal symbol is more useful when one works with manifolds more complicated than $S^{1}$. 
Now, define the sets of smooth maps

$$
S\left(S^{1}, \mathbb{C}^{k}\right)=\bigcup_{o \in \mathbb{R}} S^{o}\left(S^{1}, \mathbb{C}^{k}\right)
$$

with

$$
\begin{aligned}
& S^{o}\left(S^{1}, \mathbb{C}^{k}\right)= \\
& =\left\{\sigma \in C^{\infty}\left(T^{*} S^{1}, M_{n}(\mathbb{C})\right) \text { such that for all }(\alpha, \beta) \in \mathbb{N}^{2}:\right. \\
& \left.\qquad\left\|D_{x}^{\alpha} D_{\xi}^{\beta} \sigma(x, \xi)\right\| \leqslant C_{\alpha, \beta}(1+|\xi|)^{o-\beta}\right\}
\end{aligned}
$$

and

$$
S^{-\infty}\left(S^{1}, \mathbb{C}^{k}\right)=\bigcap_{o \in \mathbb{R}} S^{o}\left(S^{1}, \mathbb{C}^{k}\right) .
$$

The set $S\left(S^{1}, \mathbb{C}^{k}\right) / S^{-\infty}\left(S^{1}, \mathbb{C}^{k}\right)$ can be understood as the set of asymptotic expansions when $\xi \rightarrow \pm \infty$ up to rapidly decreasing maps in the $\xi$ variable. Then, we define the following multiplication rule some equivalence classes of maps $\sigma$ and $\sigma^{\prime}$ in $S\left(S^{1}, \mathbb{C}^{k}\right) / S^{-\infty}\left(S^{1}, \mathbb{C}^{k}\right)$ :

$$
\sigma \circ \sigma^{\prime}=\sum_{(\alpha) \in \mathbb{N}} \frac{(-i)^{\alpha}}{\alpha !} D_{\xi}^{\alpha} \sigma D_{x}^{\alpha} \sigma^{\prime} .
$$

Notations. We note by $C l\left(S^{1}, \mathbb{C}^{k}\right)$ classical pseudo-differential operators acting on smooth sections of $E$, and by $C l^{o}\left(S^{1}, \mathbb{C}^{k}\right)$ the space of classical pseudo-differential operators of order $o$.

There is no isomorphism between the set of symbols and the set of pseudo-differential operators. Notice that the intersection

$$
C l^{-\infty}\left(S^{1}, \mathbb{C}^{k}\right)=\bigcap_{o \in \mathbb{Z}} C l^{o}\left(S^{1}, \mathbb{C}^{k}\right)
$$

is a two-sided ideal of $C l\left(S^{1}, \mathbb{C}^{k}\right)$, and we define the quotient algebra

$$
\mathcal{F} C l\left(S^{1}, \mathbb{C}^{k}\right)=C l\left(S^{1}, \mathbb{C}^{k}\right) / C l^{-\infty}\left(S^{1}, \mathbb{C}^{k}\right),
$$

called the algebra of formal pseudo-differential operators. That algebra $\mathcal{F} C l\left(S^{1}, \mathbb{C}^{k}\right)$ is isomorphic to the set of formal symbols [4], and the identification is a morphism of $\mathbb{C}$-algebras, for the multiplication on formal symbols defined before.

2.2. Renormalized traces. Assume that $E$ is equipped this an Hermitian products $\langle\cdot, \cdot\rangle$, which induces the following $L^{2}$-inner product on sections of E:

where $d x$ is the Riemannian volume.

$$
\forall u, v \in C^{\infty}\left(S^{1}, E\right), \quad(u, v)_{L^{2}}=\int_{S^{1}}\langle u(x), v(x)\rangle d x
$$


Definition 2.3. An operation $Q$ is a weight of order $s>0$ on $E$ if and only if $Q$ is a classical, elliptic, self-adjoint, positive pseudo-differential operator acting on smooth sections of $E$.

Recall that, under these assumptions, the weight $Q$ has a real discrete spectrum, and that all its eigenspaces are finite dimensional. For such a weight $Q$ of order $q$, one can define the complex powers of $Q$, [38], see e.g. [5] for a fast overview of technicalities. The powers $Q^{-s}$ of the weight $Q$ are defined for $\operatorname{Re}(s)>0$ using a contour integral,

$$
Q^{-s}=\int_{\Gamma} \lambda^{s}(Q-\lambda \mathrm{Id})^{-1} d \lambda
$$

where $\Gamma$ is a contour around the real positive axis. Let $A$ be a $\log$ polyhomogeneous pseudo-differential operator. The map

$$
\zeta(A, Q, s)=s \in \mathbb{C} \mapsto \operatorname{tr}\left(A Q^{-s}\right) \in \mathbb{C},
$$

defined for $\operatorname{Re}(s)$ large, extends on $\mathbb{C}$ to a meromorphic function [17]. When $A$ is classical, $\zeta(A, Q, \cdot)$ has a simple pole at 0 with residue $\frac{1}{q} \operatorname{res}_{W} A$, where res $_{W}$ is the Wodzicki residue ([40], see also [11]). Notice that the Wodzicki residue extends the Adler trace [3] on formal symbols. Following textbooks $[32,37]$ for the renormalized trace of classical operators, we define

Definition 2.4. Let $A$ be a log-polyhomogeneous pseudo-differential operator. The finite part of $\zeta(A, Q, s)$ at $s=0$ is called the renormalized trace $\operatorname{tr}^{Q} A$. If $A$ is a classical pseudo-differential operator,

$$
\operatorname{tr}^{Q} A=\lim _{s \rightarrow 0}\left(\operatorname{tr}\left(A Q^{-s}\right)-\frac{1}{q s} \operatorname{res}_{W}(A)\right) .
$$

If $A$ is trace class acting on $L^{2}\left(S^{1}, \mathbb{C}^{k}\right), \operatorname{tr}^{Q}(A)=\operatorname{tr}(A)$. The functional $\operatorname{tr}^{Q}$ is of course not a trace. In this formula, it appears that the Wodzicki residue is $\operatorname{res}_{W}(A)$.

Proposition 2.5. (1) The Wodzicki residue res $_{W}$ is a trace on the algebra of classical pseudo-differential operators $C l\left(S^{1}, E\right)$, i.e. for all $A, B \in C l\left(S^{1}, V\right), \operatorname{res}_{W}[A, B]=0$.

(2) (local formula for the Wodzicki residue) Moreover, if $A \in C l\left(S^{1}, V\right)$,

$$
\operatorname{res}_{W} A=\frac{1}{2 \pi} \int_{S^{1}} \int_{|\xi|=1} \operatorname{tr} \sigma_{-1}(x, \xi) d \xi d x=\frac{1}{2 \pi} \sum_{\xi= \pm 1} \int_{S^{1}} \operatorname{tr} \sigma_{-1}(x, \xi) d \xi d x .
$$

In particular, res $W$ does not depend on the choice of $Q$.

Since $\operatorname{tr}^{Q}$ is a linear extension of the classical trace tr of trace-class operators acting on $L^{2}(S, V)$, it has weaker properties. Let us summarize some 
of them which are of interest for our work following first [5], completed by [23] for the third point.

Proposition 2.6. - Given two (classical) pseudo-differential operators $A$ and $B$, given a weight $Q$,

$$
\operatorname{tr}^{Q}[A, B]=-\frac{1}{q} \operatorname{res}(A[B, \log Q]) .
$$

- Given a differentiable family $A_{t}$ of pseudo-differential operators, given a differentiable family $Q_{t}$ of weights ofconstant order $q$,

$$
\frac{d}{d t}\left(\operatorname{tr}^{Q_{t}} A_{t}\right)=\operatorname{tr}^{Q_{t}}\left(\frac{d}{d t} A_{t}\right)-\frac{1}{q} \operatorname{res}\left(A_{t}\left(\frac{d}{d t} \log Q_{t}\right)\right) .
$$

- Under the previous notations, if $C$ is a classical elliptic injective operator or a diffeomorphism, $\operatorname{tr}^{C^{-1} Q C}\left(C^{-1} A C\right)$ is well-defined and equals $\operatorname{tr}^{Q} A$.

- Finally,

$$
\operatorname{tr}^{Q} A=\overline{\operatorname{tr}^{*} A^{*}} .
$$

Since $\operatorname{tr}^{Q}$ is not tracial, let us give more details on the renormalized trace of the bracket, following [19].

Definition 2.7. Let $E$ be a vector bundle over $S^{1}$, let $Q$ a weight and let $a \in \mathbb{Z}$. We define:

$$
\mathcal{A}_{a}^{Q}=\left\{B \in C l\left(S^{1}, E\right) \mid[B, \log Q] \in C l^{a}\left(S^{1}, E\right)\right\} .
$$

Theorem 2.8. $\quad$ (i) $\mathcal{A}_{a}^{Q} \cap C l^{0}\left(S^{1}, E\right)$ is an subalgebra of $C l\left(S^{1}, E\right)$ with unit.

(ii) Let $B \in \operatorname{Ell}^{*}\left(S^{1}, E\right), B^{-1} \mathcal{A}_{a}^{Q} B=\mathcal{A}_{a}^{B^{-1} Q B}$.

(iii) Let $A \in C l^{b}\left(S^{1}, E\right)$, and $B \in \mathcal{A}_{-b-2}^{Q}$, then $\operatorname{tr}^{Q}[A, B]=0$. As a consequence,

$$
\forall(A, B) \in C l^{-\infty}\left(S^{1}, V\right) \times C l\left(S^{1}, V\right), \quad \operatorname{tr}^{Q}[A, B]=0 .
$$

When needed and appropriate, other properties of renormalized traces will be given later.

\subsection{Splittings of $\mathcal{F} C l\left(S^{1}, V\right)$.}

2.9.1. The polarization operator. The operator $D=-i \frac{d}{d x} \operatorname{splits} C^{\infty}\left(S^{1}, \mathbb{C}^{n}\right)$ into three spaces:

- its kernel $E_{0}$, built of constant maps

- $E_{+}$, the vector space spanned by eigenvectors related to positive eigenvalues 
- $E_{-}$, the vector space spanned by eigenvectors related to negative eigenvalues.

The $L^{2}$-orthogonal projection on $E_{0}$ is a smoothing operator, which has null formal symbol. By the way, concentrating our attention on the formal symbol of operators first, we can ignore this projection and hence we work on $E_{+} \oplus E_{-}$. The following elementary result will be useful for the sequel.

Lemma 2.10. $[18,21]$

(1) $\sigma(D)=\xi$.

(2) $\sigma(|D|)=|\xi|$.

(3) $\sigma(\epsilon)=\frac{\xi}{|\xi|}$, where $\epsilon=D|D|^{-1}=|D|^{-1} D$ is the sign of $D$.

(4) Let $p_{E_{+}}\left(\right.$resp. $\left.p_{E_{-}}\right)$be the projection on $E_{+}$(resp. E- $\left.E_{-}\right)$, then

$$
\sigma\left(p_{E_{+}}\right)=\frac{1}{2}\left(\operatorname{Id}+\frac{\xi}{|\xi|}\right), \quad \sigma\left(p_{E_{-}}\right)=\frac{1}{2}\left(\operatorname{Id}-\frac{\xi}{|\xi|}\right) .
$$

Remark 2.11. The operators $D$ and $\epsilon$ are operators acting on $L^{2}\left(S^{1}, \mathbb{C}\right)$ while $\frac{d}{d x}$ and $i \epsilon$ are operators on $L^{2}\left(S^{1}, \mathbb{C}\right)$ which leave invariant $L^{2}\left(S^{1}, \mathbb{R}\right)$.

Let us now give a trivial but very useful lemma:

Lemma 2.12 ([18]). Let $f: \mathbb{R}^{*} \rightarrow V$ be a 0-positively homogeneous function with values in a topological vector space $V$. Then, for any $n \in \mathbb{N}^{*}$, $f^{(n)}=0$ where $f^{(n)}$ denotes the $n$-th derivative of $f$.

Corollary 2.13. Let $A \in \mathcal{F} C l\left(S^{1}, \mathbb{C}^{n}\right)$ such that $\sigma(A)=\sigma_{0}(A)$. Then

$$
\sigma(A \circ B)=\sigma_{0}(A) \cdot \sigma(B) \text { (pointwise multiplication). }
$$

2.13.1. The splitting with induced by the connected components of $T^{*} S^{1}-S^{1}$. In this section, we define two ideals of the algebra $\mathcal{F} C l\left(S^{1}, V\right)$, that we call $\mathcal{F} C l_{+}\left(S^{1}, V\right)$ and $\mathcal{F} C l_{-}\left(S^{1}, V\right)$, such that

$$
\mathcal{F} C l\left(S^{1}, V\right)=\mathcal{F} C l_{+}\left(S^{1}, V\right) \oplus \mathcal{F} C l_{-}\left(S^{1}, V\right) .
$$

This decomposition is explicit in [11, section 4.4., p. 216], and we give an explicit description here following [18,21].

Definition 2.14. Let $\sigma$ be a partial symbol of order o on E. Then, we define, for $(x, \xi) \in T^{*} S^{1}-S^{1}$,

$$
\sigma_{+}(x, \xi)=\left\{\begin{array}{ll}
\sigma(x, \xi) & \text { if } \xi>0 \\
0 & \text { if } \xi<0
\end{array} \text { and } \sigma_{-}(x, \xi)= \begin{cases}0 & \text { if } \xi>0 \\
\sigma(x, \xi) & \text { if } \xi<0\end{cases}\right.
$$

We define $p_{+}(\sigma)=\sigma_{+}$and $p_{-}(\sigma)=\sigma_{-}$. 
The maps

$$
\begin{aligned}
& p_{+}: \mathcal{F} C l\left(S^{1}, V\right) \rightarrow \mathcal{F} C l\left(S^{1}, V\right), \\
& p_{-}: \mathcal{F} C l\left(S^{1}, V\right) \rightarrow \mathcal{F} C l\left(S^{1}, V\right)
\end{aligned}
$$

are clearly smooth algebra morphisms (yet non-unital morphisms) that leave the order invariant and are also projections (since multiplication on formal symbols is expressed in terms of pointwise multiplication of tensors).

Definition 2.15. We define

$$
\begin{aligned}
& \mathcal{F} C l_{+}\left(S^{1}, V\right)=\operatorname{Im}\left(p_{+}\right)=\operatorname{Ker}\left(p_{-}\right), \\
& \mathcal{F} C l_{-}\left(S^{1}, V\right)=\operatorname{Im}\left(p_{-}\right)=\operatorname{Ker}\left(p_{+}\right) .
\end{aligned}
$$

Since $p_{+}$is a projection, we have the splitting

$$
\mathcal{F} C l\left(S^{1}, V\right)=\mathcal{F} C l_{+}\left(S^{1}, V\right) \oplus \mathcal{F} C l_{-}\left(S^{1}, V\right) .
$$

Let us give another characterization of $p_{+}$and $p_{-}$. Looking more precisely at the formal symbols of $p_{E_{+}}$and $p_{E_{-}}$computed in Lemma 2.10, we observe that

$$
\sigma\left(p_{E_{+}}\right)=\left\{\begin{array}{ll}
1 & \text { if } \xi>0, \\
0 & \text { if } \xi<0,
\end{array} \quad \text { and } \quad \sigma\left(p_{E_{-}}\right)= \begin{cases}0 & \text { if } \xi>0 \\
1 & \text { if } \xi<0\end{cases}\right.
$$

In particular, we have that $p_{+}$and $p_{-}$satisfy Corollary 2.13. Moreover, their symbol do not depend on $x$. From this, we have the following result.

Proposition 2.16. Let $A \in \mathcal{F} C l\left(S^{1}, V\right)$. Then

$$
\begin{aligned}
& p_{+}(A)=\sigma\left(p_{E_{+}}\right) \circ A=A \circ \sigma\left(p_{E_{+}}\right), \\
& p_{-}(A)=\sigma\left(p_{E_{-}}\right) \circ A=A \circ \sigma\left(p_{E_{-}}\right) .
\end{aligned}
$$

2.16.1. The "odd-even" splitting. Denote by $\sigma(A)(x, \xi)$ the total formal symbol of $A \in \mathcal{F} C l\left(S^{1}, V\right)$. The following proposition is trivial:

Proposition 2.17. Define $\phi: \mathcal{F} C l\left(S^{1}, V\right) \rightarrow \mathcal{F} C l\left(S^{1}, V\right)$ by

$$
\phi(A)=\frac{1}{2} \sum_{k \in \mathbb{Z}} \sigma_{k}(A)(x, \xi)-(-1)^{k} \sigma_{k}(A)(x,-\xi) .
$$

Then this map is smooth, and

$$
\Psi D O\left(S^{1}, V\right)=\mathcal{F} C l_{e e}\left(S^{1}, V\right)=\operatorname{Ker}(\phi) .
$$


Following [37], one can define the even-odd class pseudo-differential operators

$$
\begin{aligned}
& \mathcal{F} C l_{\text {eo }}(\left.S^{1}, V\right)= \\
& \quad=\left\{A \in \mathcal{F} C l\left(S^{1}, V\right) \mid \sum_{k \in \mathbb{Z}} \sigma_{k}(A)(x, \xi)+(-1)^{k} \sigma_{k}(A)(x,-\xi)=0\right\} .
\end{aligned}
$$

Remark 2.18. In e.g. [13,14], even-even class pseudodifferential operators are called odd class pseudodifferential operators. By the way, following the terminology of $[13,14]$ even-odd class pseudo-differential operators should be called even class. In this paper we prefer to fit with the terminology given in the textbooks $[32,37]$.

Proposition 2.19. $\phi$ is a projection and $\mathcal{F} C l_{e o}\left(S^{1}, V\right)=\operatorname{Im} \phi$.

By the way, we also have

$$
\mathcal{F} C l\left(S^{1}, V\right)=\mathcal{F} C l_{e e}\left(S^{1}, V\right) \oplus \mathcal{F} C l_{e o}\left(S^{1}, V\right) .
$$

We have the following composition rules for the class of a formal operator $A \circ B$ :

\begin{tabular}{|c|c|c|}
\hline & $A$ even-even class & $A$ even class \\
\hline$B$ even-even class & $A \circ B$ even-even class & $A \circ B$ even-odd class \\
\hline$B$ even-odd class & $A \circ B$ even-odd class & $A \circ B$ even-even class \\
\hline
\end{tabular}

Example 2.20. $\epsilon(D)$ and $|D|$ are even-odd class, while we already mentioned that differential operators are even-even class.

Applying the local formula for Wodzicki residue, one can prove [5]:

Proposition 2.21. If $A$ and $Q$ lie in the odd class, then $f(s)=\operatorname{tr}\left(A Q^{-s}\right)$ has no pole at $s=0$. Moreover, if $A$ and $B$ are odd class pseudo-differential operators, $\operatorname{tr}^{Q}([A, B])=0$ and $\operatorname{tr}^{Q} A$ does not depend on $Q$.

\subsection{Formal and non-formal $\operatorname{Diff}\left(S^{1}\right)$-pseudodifferential operators.} It follows from $[6,31]$ that the group $\operatorname{Diff}_{+}\left(S^{1}\right)$ is open in the Fréchet manifold $C^{\infty}\left(S^{1}, S^{1}\right)$. This fact makes it a Fréchet manifold and, following [31], a regular Fréchet Lie group. In addition to groups of pseudo-differential operators, we also need a restricted class of groups of Fourier integral operators which we will call $\operatorname{Diff}\left(S^{1}\right)$-pseudodifferential operators following [23, 25]. These groups appear as central extensions of $\operatorname{Diff}\left(S^{1}\right)$ or $\operatorname{Diff}_{+}\left(S^{1}\right)$ by groups of pseudo-differential operators. We do not state the basic facts on Fourier integral operators here (they can be found in the classical paper [9]). The pseudo-differential operators considered here can be classical, 
odd class, or anything else. Applying the formulas of "changes of coordinates" (which can be understood as adjoint actions of diffeomorphisms) of e.g. [8], we obtain that even-even and even-odd class pseudo-differential operators are stable under the adjoint action of $\operatorname{Diff}\left(S^{1}\right)$. Thus, we can define the following groups [23]:

Definition 2.23. 1) The group $\mathcal{F} C l_{\operatorname{Diff}\left(S^{1}\right)}^{*}\left(S^{1}, V\right)$ is an infinite dimensional group defined by $\mathcal{F} C l_{\text {Diff }\left(S^{1}\right)}^{*}\left(S^{1}, V\right)=\left\{A=B \circ g \mid B \in C l^{*}\left(S^{1}, V\right)\right.$ and $\left.g \in \operatorname{Diff}\left(S^{1}\right)\right\}$.

2) The group $\mathcal{F} C l_{\operatorname{Diff}\left(S^{1}\right)}^{0, *}\left(S^{1}, V\right)$ is an infinite dimensional group defined by $\mathcal{F} C l_{\operatorname{Diff}\left(S^{1}\right)}^{0, *}\left(S^{1}, V\right)=\left\{A=B \circ g \mid B \in C l^{0, *}\left(S^{1}, V\right)\right.$ and $\left.g \in \operatorname{Diff}\left(S^{1}\right)\right\}$.

3) The group $\mathcal{F} C l_{\mathrm{Diff}\left(S^{1}\right), e e}^{*}\left(S^{1}, V\right)$ is an infinite dimensional group defined by

$$
\mathcal{F} C l_{\text {Diff }\left(S^{1}\right), e e}^{*}\left(S^{1}, V\right)=\left\{A=B \circ g \mid B \in C l_{e e}^{*}\left(S^{1}, V\right) \text { and } g \in \operatorname{Diff}\left(S^{1}\right)\right\} \text {. }
$$

4) The group $\mathcal{F} C l_{\operatorname{Diff}\left(S^{1}\right), e e}^{0, *}\left(S^{1}, V\right)$ is an infinite dimensional group defined by

$$
\mathcal{F} C l_{\operatorname{Diff}\left(S^{1}\right), e e}^{0, *}\left(S^{1}, V\right)=\left\{A=B \circ g \mid B \in C l_{e e}^{0, *}\left(S^{1}, V\right) \text { and } g \in \operatorname{Diff}\left(S^{1}\right)\right\} .
$$

Remark 2.24. This construction of phase functions of $\operatorname{Diff}\left(S^{1}\right)$-pseudodifferential operators differs from the one described by Omori [31] and Adams, Ratiu and Schmid [1] for some groups of Fourier integral operators; the exact relation among these constructions still needs to be investigated.

Remark 2.25. The decomposition $A=B \circ g$ is unique [23], and the diffeomorphism appears as the phase of the Fourier integral operator.

Remark 2.26. The group $\operatorname{Diff}\left(S^{1}\right)$ decomposes into two connected components $\operatorname{Diff}\left(S^{1}\right)=\operatorname{Diff}_{+}\left(S^{1}\right) \cup \operatorname{Diff}_{-}\left(S^{1}\right)$, where the connected component of the identity, Diff $+\left(S^{1}\right)$, is the group of orientation preserving diffeomorphisms of $S^{1}$. By the way, we can replace $\operatorname{Diff}\left(S^{1}\right)$ by $\operatorname{Diff}_{+}\left(S^{1}\right)$ in the previous definition.

Remark 2.27. We have, on these Lie groups, some difficulties to exhibit an atlas especially when considering unbounded operators. One then can consider "natural" notions of smoothness, inherited from the embedding into $C l\left(S^{1}, V\right)$ for the pseudo-differential part, and from the well-known structure of ILB Lie group [31] from the diffeomorphism (phase) component. In order to be more rigorous, one can then consider Frölicher Lie groups along the lines of $[23,25]$ in this context, or in $[24,27]$ when dealing with 
other examples where this setting is useful. A not-so-complete description of technical properties of Frölicher Lie groups can be found in works by other authors $[16,29,30]$ but this area of knowledge, however, still needs to be further developed. This lack of theoretical knowledge is not a problem for understanding and dealing with groups of $\operatorname{Diff}\left(S^{1}\right)$-pseudo-differential operators.

From this remark, we deduce that the Lie algebra of $\mathcal{F} C l_{\operatorname{Diff}\left(S^{1}\right)}^{*}\left(S^{1}, V\right)$ is $C l\left(S^{1}, V\right) \oplus \operatorname{Vect}\left(S^{1}\right)$, and we note that

$$
(a, X) \mapsto a+X
$$

is a Lie algebra morphism with values in $C l\left(S^{1}, V\right)$. The same remark holds for subgroups of $\mathcal{F} C l_{\text {Diff }\left(S^{1}\right)}^{*}\left(S^{1}, V\right)$. Let us now define a relation of equivalence "up to smoothing operators".

Definition 2.28. Let $\left(A, A^{\prime}\right) \in\left(\mathcal{F} C l_{\text {Diff }\left(S^{1}\right)}^{*}\left(S^{1}, V\right)\right)^{2}$, with $A=B \circ g$ and $A^{\prime}=B^{\prime} \circ g^{\prime}$ as before. Then

$$
A \equiv A^{\prime} \quad \Leftrightarrow \quad\left\{\begin{array}{l}
g=g^{\prime} \\
B-B^{\prime} \in C l^{-\infty}\left(S^{1}, V\right) .
\end{array}\right.
$$

The set of equivalence classes with respect to $\equiv$ is denoted by

$$
\mathcal{F} F C l_{\operatorname{Diff}\left(S^{1}\right)}^{*}\left(S^{1}, V\right)
$$

and is called the set of formal $\operatorname{Diff}\left(S^{1}\right)$-pseudodifferential operators.

The same spaces of formal operators can be constructed using orientationpreserving diffeomorphisms of $S^{1}$, even-even class pseudodifferential operators and so on. We do not feel the need to give here redundant constructions, and obvious notations.

Theorem 2.29. Let

$$
G=\left\{A \in C l^{0 ; *}\left(S^{1}, V\right) \mid A=\mathrm{Id}+B, B \in C l^{-\infty}\left(S^{1}, V\right)\right\} .
$$

Then

- $G$ is a normal subgroup of $\mathcal{F} C l_{\operatorname{Diff}\left(S^{1}\right)}^{*}\left(S^{1}, V\right)$, i.e.

$$
G \triangleleft \mathcal{F} C l_{\operatorname{Diff}\left(S^{1}\right)}^{*}\left(S^{1}, V\right),
$$

- given $\left(A, A^{\prime}\right) \in \mathcal{F} C l_{\operatorname{Diff}\left(S^{1}\right)}^{*}\left(S^{1}, V\right)^{2}$,

$$
A \equiv A^{\prime} \quad \Longleftrightarrow \quad A A^{\prime-1} \in G
$$


which implies that

$$
\mathcal{F} F C l_{\operatorname{Diff}\left(S^{1}\right)}^{*}\left(S^{1}, V\right)=\mathcal{F} C l_{\operatorname{Diff}\left(S^{1}\right)}^{*}\left(S^{1}, V\right) / G .
$$

By the way, $\mathcal{F} F C l_{\operatorname{Diff}\left(S^{1}\right)}^{*}\left(S^{1}, V\right)$ is a group. Moreover,

$$
\mathcal{F} F C l_{\operatorname{Diff}\left(S^{1}\right)}^{*}\left(S^{1}, V\right)=\mathcal{F} C l^{*}\left(S^{1}, V\right) \rtimes \operatorname{Diff}\left(S^{1}\right) .
$$

Proof. Let $\left(A, A^{\prime}\right) \in F C l_{\operatorname{Diff}\left(S^{1}\right)}^{*}\left(S^{1}, V\right)$ with $A=B \circ g, A^{\prime}=B^{\prime} \circ g^{\prime}$ in the decomposition $F C l_{\operatorname{Diff}\left(S^{1}\right)}^{*}\left(S^{1}, V\right)=C l^{*}\left(S^{1}, V\right) \rtimes \operatorname{Diff}\left(S^{1}\right)$. Then the following conditions are equivalent:
(a) $A \equiv A^{\prime}$
(b) $\left\{\begin{array}{l}g=g^{\prime} \\ B-B^{\prime}=-R \in C l^{-\infty}\left(S^{1}, V\right)\end{array}\right.$
(c) $A A^{\prime-1}=B B^{\prime-1}=\mathrm{Id}+R B^{\prime-1} \in G$.

Let $A \in F C l_{\operatorname{Diff}\left(S^{1}\right)}^{*}\left(S^{1}, V\right)$ and let $\operatorname{Id}+S \in G$ with $S \in C l^{-\infty}\left(S^{1}, \mathbb{C}\right)$. Then

$$
A^{-1}(\mathrm{Id}+S) A=\mathrm{Id}+A^{-1} S A .
$$

We have $A=B \circ g$ with $B \in C l^{*}\left(S^{1}, V\right)$ and $g \in \operatorname{Diff}\left(S^{1}\right)$. As an operator acting on $L^{2}\left(S^{1}, V\right)$-functions, composition on the right by a diffeomorphism is a bounded operator. As an operator from $C^{\infty}\left(S^{1}, V\right)$ to $C^{\infty}\left(S^{1}, V\right)$, composition on the right by a diffeomorphism is also a bounded operator. A pseudo-differential operator of order $o$ is a bounded operator from $L^{2}\left(S^{1}, V\right)$ to $H^{-o}\left(S^{1}, V\right)$, and also from $C^{\infty}\left(S^{1}, V\right)$ to $C^{\infty}\left(S^{1}, V\right)$. Finally a smoothing operator is bounded from any Sobolev space $H^{-o}$ to $C^{\infty}$. By the way,

$$
A^{-1} S A=g^{-1} B^{-1} S B g \in C l^{-\infty}\left(S^{1}, V\right) .
$$

Hence $G \triangleleft \mathcal{F} C l_{\operatorname{Diff}\left(S^{1}\right)}^{*}\left(S^{1}, V\right)$. Let us now examine the following diagram:

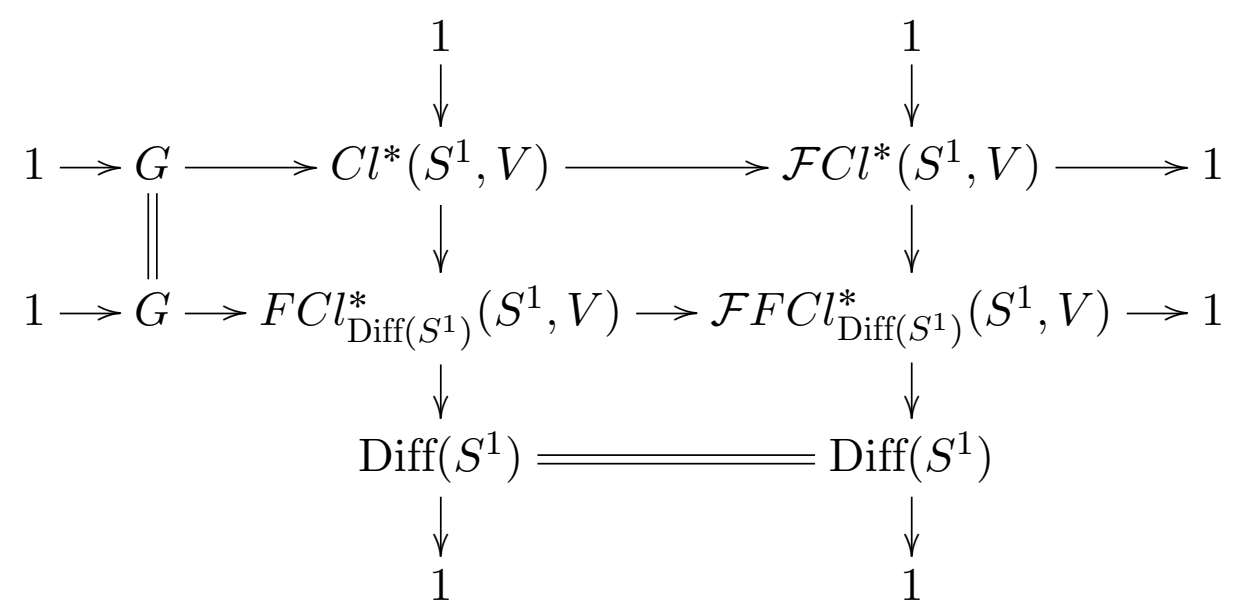


The three squares commute, the two horizontal lines are short exact sequences as well as the central column. By the way, we also have that

$$
1 \rightarrow \mathcal{F} C l^{*}\left(S^{1}, V\right) \rightarrow \mathcal{F} F C l_{\operatorname{Diff}\left(S^{1}\right)}^{*}\left(S^{1}, V\right) \rightarrow \operatorname{Diff}\left(S^{1}\right) \rightarrow 1
$$

is a short exact sequence.

Via identification $(2.4), \mathcal{F} F C l_{\operatorname{Diff}\left(S^{1}\right)}^{*}\left(S^{1}, V\right)$ as a (set theoretic) product can be equiped by the product topology of $\mathcal{F} C l^{*}\left(S^{1}, V\right) \times \operatorname{Diff}\left(S^{1}\right)$. This makes of it a smooth Lie group modeled on a locally convex topological vector space, and we can state:

Proposition 2.30. There is a natural structure of infinite dimensional Lie group on $\mathcal{F} F C l_{\operatorname{Diff}\left(S^{1}\right)}^{*}\left(S^{1}, V\right)$, and its Lie algebra (defined by germs of smooth paths) reads as

$$
\mathcal{F} C l\left(S^{1}, V\right) \rtimes \operatorname{Vect}\left(S^{1}\right) .
$$

Remark 2.31. It is proven in [23] that, in an algebra of formal pseudodifferential operators that can be identified with $\mathcal{F} C l_{e e}\left(S^{1}, \mathbb{C}\right)$ in our context, the constant vector field $t \mapsto \frac{d}{d x}$ does not integrate to a smooth path on the group, in other words

$$
\left.\exp \left(\frac{d}{d x}\right) \notin \mathcal{F} C l_{e e}^{*}\left(S^{1}, \mathbb{C}\right)\right) .
$$

This shows that $\mathcal{F} C l_{\text {ee }}^{*}\left(S^{1}, \mathbb{C}\right)$, and therefore $C l^{*}\left(S^{1}, V\right)$ and also the space $F C l_{\text {Diff }\left(S^{1}\right)}^{*}\left(S^{1}, V\right)$ are not regular in the sense of Omori [31], while the same constant vector, understood as an element of $\operatorname{Vect}\left(S^{1}\right)$, the Lie algebra of $\operatorname{Diff}\left(S^{1}\right)$, integrates to a rotation on the circle. This shows that one has to be careful on which component the differential monomials of degree 1 are considered while working with $F C l_{\text {Diff }\left(S^{1}\right)}^{*}\left(S^{1}, V\right)$. Its Lie algebra cannot be embedded but only represented in $C l_{e e}\left(S^{1}, V\right)$.

\section{Relation With the Restricted Linear Group}

3.1. On cocycles on $C l\left(S^{1}, \mathbb{C}^{k}\right)$. Let us first precise which polarization we choose on $L^{2}\left(S^{1}, \mathbb{C}^{k}\right)$. We can choose independently two polarizations:

- one setting $H_{+}^{(1)}=E_{+}$and $H_{-}^{(1)}=E_{0} \oplus E_{-}$,

- or another one setting $H_{+}^{(2)}=E_{+} \oplus E_{0}$ and $H_{-}^{(2)}=E_{-}$.

Since $E_{0}$ is of dimension $k$, the orthogonal projection on $E_{0}$ is a smoothing operator. Hence,

$$
\sigma\left(p_{H_{+}^{(1)}}\right)=\sigma\left(p_{H_{+}^{(2)}}\right)=1_{\xi>0}
$$


and

$$
\sigma\left(p_{H_{-}^{(1)}}\right)=\sigma\left(p_{H_{-}^{(2)}}\right)=1_{\xi<0}
$$

We introduce the notation, for $A \in P D O\left(S^{1}, \mathbb{C}^{k}\right)$,

$$
A_{++}=p_{H_{+}} A p_{H_{+}},
$$

where $H_{+}$denotes $H_{+}^{(1)}$ or $H_{+}^{(2)}$, and we set $\epsilon(D)=p_{H_{+}}-p_{H_{-}}$. We notice that $\sigma\left(A_{++}\right)=\sigma_{+}(A)$, and recall the following result [21]:

Theorem 3.2. For any $A \in C l\left(S^{1}, \mathbb{C}^{k}\right),[A, \epsilon(D)] \in C l^{-\infty}\left(S^{1}, \mathbb{C}^{k}\right)$. Consequently,

$$
c_{s}^{D}: A, B \in C l\left(S^{1}, E\right) \mapsto \frac{1}{2} \operatorname{tr}(\epsilon(D)[\epsilon(D), A][\epsilon(D), B])=\operatorname{tr}([A, \epsilon(D)] B)
$$

is a well-defined 2-cocycle on $\operatorname{PDO}\left(S^{1}, \mathbb{C}^{k}\right)$. Moreover, $c_{s}^{D}$ is non trivial on any Lie algebra $\mathcal{A}$ such that $C^{\infty}\left(S^{1}, \mathbb{C}^{k}\right) \subset \mathcal{A} \subset C l\left(S^{1}, \mathbb{C}^{k}\right)$.

Along this cocycle, we have to mention two others. First, the KravchenkoKhesin cocycle [15], defined on Adler series $a=\sum_{n \leqslant k} a_{n} \xi^{n}$ and $b=\sum_{m \leqslant l} b_{m} \xi^{m}$ by

$$
c_{K K}(a, b)=\operatorname{res}(a[b, \log \xi])
$$

which pulls-back on the algebra $C l\left(S^{1}, \mathbb{C}^{k}\right)$, following a procedure first described by Radul [35] to our knowledge, to a cocycle called KravchenkoKhesin-Radul cocycle in [20], defined in its polarized version by

$$
c_{K K R}(A, B)=\operatorname{tr}^{|D|}\left(\left[A_{++}, B_{++}\right]\right)=\frac{1}{2 \pi} \operatorname{res}_{W}\left(A_{++}\left[B_{++}, \log |D|\right]\right),
$$

which is the pull-back, up to the constant $\frac{1}{2 \pi}$, of $c_{K K}$ through the map

$$
A \in C l\left(S^{1}, \mathbb{C}^{k}\right) \mapsto \sigma_{+}(A) .
$$

Secondly, the index cocycle described in [34] and extended to $C l\left(S^{1}, \mathbb{C}^{k}\right)$ in [21], defined by:

$$
\lambda(A, B)=\operatorname{tr}\left(\left[A_{++}, B_{++}\right]-[A, B]_{++}\right) .
$$

We have to remark that, in order to have a well-defined formula, the operator $\left[A_{++}, B_{++}\right]-[A, B]_{++}$needs to be trace-class. When $A$ and $B$ are pseudo-differential operators, this operator is smoothing and hence the trace is well-defined. Following [21], we can state:

Proposition 3.3. On $\operatorname{PDO}\left(S^{1}, \mathbb{C}^{k}\right)$,

$$
\lambda=\frac{1}{2} c_{s} .
$$

Moreover, on any algebra $\mathcal{A}$ such that $C^{\infty}\left(S^{1}, \mathbb{C}^{k}\right) \subset \mathcal{A} \subset C l\left(S^{1}, \mathbb{C}^{k}\right)$, the cocycles $c_{K K R}, \lambda$ and $\frac{1}{2} c_{s}$ are non trivial in Hoschild cohomology, and belong to the same cohomology class. 
3.4. $\mathrm{GL}_{\mathrm{res}}$ and its subgroups of Fourier-integral operators. Let us now turn to the Lie group of bounded operators described in [34]:

$$
\begin{aligned}
\operatorname{GL}_{\mathrm{res}}\left(S^{1}, \mathbb{C}^{k}\right)= \\
\quad=\left\{u \in \operatorname{GL}\left(L^{2}\left(S^{1}, \mathbb{C}^{k}\right)\right) \text { such that }[\epsilon(D), u] \text { is Hilbert-Schmidt }\right\}
\end{aligned}
$$

with Lie algebra

$$
\mathcal{L}\left(S^{1}, \mathbb{C}^{k}\right)=\left\{u \in \mathcal{L}\left(L^{2}\left(S^{1}, \mathbb{C}^{k}\right)\right) \text { such that }[\epsilon(D), u] \text { is Hilbert-Schmidt }\right\} .
$$

Proposition 3.5. ([23, Theorems 25-26])

$$
F C l_{\operatorname{Diff}_{+}\left(S^{1}\right)}^{0, *}\left(S^{1}, \mathbb{C}^{k}\right) \subset \operatorname{GL}_{\mathrm{res}}\left(S^{1}, \mathbb{C}^{k}\right) .
$$

Let us now give a new light on an old result present in [34] from a topological viewpoint, expressed by remarks but not stated clearly in the mathematical literature to our knowledge. We choose here a new approach for the proof, more easy and much more fast, and adapted to our approach of (maybe generalized) differentiability prior to topological considerations.

Lemma 3.6. The injection map $\operatorname{Diff}_{+}\left(S^{1}\right) \hookrightarrow \mathrm{GL}_{\mathrm{res}}\left(S^{1}, \mathbb{C}^{k}\right)$ is not differentiable.

Proof. Let us assume that the injection map $\operatorname{Diff}_{+}\left(S^{1}\right) \hookrightarrow \operatorname{GL}_{\mathrm{res}}\left(S^{1}, \mathbb{C}^{k}\right)$ is differentiable. The group $\mathrm{GL}_{\mathrm{res}}\left(S^{1}, \mathbb{C}^{k}\right)$ is acting smoothly on $L^{2}\left(S^{1}, \mathbb{C}^{n}\right)$ and hence the Lie algebra of $\operatorname{GL}_{\text {res }}\left(S^{1}, \mathbb{C}^{k}\right)$ is a Lie algebra of bounded operators acting on $L^{2}\left(S^{1}, \mathbb{C}^{n}\right)$. The Lie algebra $\operatorname{Vect}\left(S^{1}\right)$ is a Lie algebra of unbounded operators acting on $L^{2}\left(S^{1}, \mathbb{C}^{n}\right)$ hence the injection map $\operatorname{Diff}_{+}\left(S^{1}\right) \hookrightarrow \mathrm{GL}_{\mathrm{res}}\left(S^{1}, \mathbb{C}^{k}\right)$ is not differentiable.

From this Lemma, next theorem is straightforward:

Theorem 3.7. The injection maps $F C l_{\operatorname{Diff}_{+}\left(S^{1}\right)}^{0 *}\left(S^{1}, \mathbb{C}^{k}\right) \hookrightarrow \mathrm{GL}_{\mathrm{res}}\left(S^{1}, \mathbb{C}^{k}\right)$ and $D O^{0, *}\left(S^{1}, \mathbb{C}^{k}\right) \rtimes \operatorname{Diff}_{+}\left(S^{1}\right) \hookrightarrow \mathrm{GL}_{\mathrm{res}}\left(S^{1}, \mathbb{C}^{k}\right)$ are not differentiable.

3.8. Index cocycle on $F C l_{\operatorname{Diff}\left(S^{1}\right)}^{0, *}\left(S^{1}, \mathbb{C}^{k}\right)$. Let us now recall a result from [34]:

Proposition 3.9. Let us consider the right-invariant 2-form generated by $\lambda$ on $\mathrm{GL}_{\mathrm{res}}$, that we note by $\tilde{\lambda}$. Then $\tilde{\lambda}$ is a closed, non exact 2 -form on $\mathrm{GL}_{\mathrm{res}}$.

Lemma 3.10. Let $\gamma: \mathbb{R} \rightarrow F C l_{\text {Diff }_{+}\left(S^{1}\right)}^{*}\left(S^{1}, \mathbb{C}^{k}\right)$ be a smooth path. Then the path

$$
t \in \mathbb{R} \longmapsto[\epsilon(D), \gamma(t)]
$$


is a smooth path of smoothing operators with respect to any of these differentiable structures:

- the differentiable structure of $F C l_{\text {Diff }_{+}\left(S^{1}\right)}\left(S^{1}, \mathbb{C}^{k}\right)$

- the differentiable structure of $\mathrm{GL}_{\mathrm{res}}\left(S^{1}, \mathbb{C}^{k}\right.$

Proof. The proof follows from

$$
\left[F C l_{\text {Diff }_{+}\left(S^{1}\right)}^{*}\left(S^{1}, \mathbb{C}^{k}\right), \epsilon(D)\right] \subset C l^{-\infty}\left(S^{1}, \mathbb{C}^{k}\right)
$$

as is stated in [23, Theorem 25].

As a consequence, we get:

Theorem 3.11. $\lambda=\frac{1}{2} c_{s}$ generates a closed, non exact 2 -form on

$$
F C l_{\text {Diff }_{+}\left(S^{1}\right)}^{*}\left(S^{1}, \mathbb{C}^{k}\right)
$$

\section{Renormalized extension of the Hilbert-Schmidt Hermitian METRIC}

The vector space $C l^{-1}\left(S^{1}, V\right)$ is a space of Hilbert-Schmidt operators. As a subspace, $\mathrm{Cl}^{-1}\left(S^{1}, V\right)$ inherits a Hermitian metric from the classical Hilbert-Schmidt inner product. The renormalized trace $\operatorname{tr}^{\Delta}$ extends the classical trace tr of trace class operators to a smooth linear functional on $C l\left(S^{1}, V\right)$. We investigate here the possible (maybe naive) extension of the classical Hilbert-Schmidt inner product to $C l\left(S^{1}, V\right)$ via $\operatorname{tr}^{\Delta}$.

4.1. Calculation of renormalized traces. Let $\left(z^{k}\right)_{k \in \mathbb{Z}}$ is the Fourier $L^{2}$-orthonormal basis. Let us recall that there exists an ambiguity on $\epsilon(D)$ concerning its action on $z^{0}$, which can be, or not, in the kernel of $p_{+}$, or in the eigenspace of the eigenvalue 1 or -1 . Depending on each of these three possibilities respectively, we set $\epsilon(k)$ as the eigenvalue of $\epsilon(D)$ at the eigenvector $z^{k}$.

Lemma 4.2. Let $X=u \frac{d}{d x}, Y=v \frac{d}{d x}$ be two vector fields over $S^{1}$, and let $a, b \in C^{\infty}\left(S^{1}, \mathbb{C}\right)$. Then

(1) $\operatorname{tr}^{\Delta}(a \bar{b})=0$,

(2) $\operatorname{tr}^{\Delta}\left(X Y^{*}\right)=0$,

(3) $\operatorname{tr}^{\Delta}(X a)=\operatorname{tr}^{\Delta}(a X)=0$.

Proof. We have here even-even class operators, so that the renormalized trace is commuting in all items. 
(1) Following computations presented e.g. in [5], we compute for $\mathfrak{R e}(s)$ large enough first, with the convention $0^{-s}=1$ :

$$
\begin{aligned}
\sum_{k \in \mathbb{Z}}\left(\left(z^{n} z^{-m} z^{k}\right), \Delta^{-s} z^{k}\right)_{L^{2}} & =\sum_{k \in \mathbb{Z}}\left(k^{2}\right)^{-s}\left(z^{n} z^{-m} z^{k}, z^{k}\right)_{L^{2}} \\
& =\sum_{k \in \mathbb{Z}}\left(k^{2}\right)^{-s}\left(z^{n-m+k}, z^{k}\right)_{L^{2}} \\
& = \begin{cases}0 & \text { if } n \neq m \\
1+2 \sum_{k \in \mathbb{N}^{*}}\left(k^{2}\right)^{-s} & \text { if } n=m .\end{cases}
\end{aligned}
$$

By the way, taking the limit as $s \rightarrow 0$ in the zeta-renormalization procedure of the trace, we get, dividing the sum for $k \in \mathbb{Z}$ into three parts: $k \in \mathbb{Z}_{-}^{*}$, $k=0$ and $k \in \mathbb{N}^{*}$ :

$$
\operatorname{tr}^{\Delta}(a \bar{b})=(\zeta(0)+1+\zeta(0))(a, b)_{L^{2}}=0(a, b)_{L^{2}} .
$$

(2) Here the functions $u$ and $v$ are real-valued, which means that one should consider the real Fourier basis for the summation. However, since the real Fourier basis is a linear combination of the complex one, we investigate first the renormalized trace with $u=z^{n}$ and $v=z^{m}$. Then

$$
X Y^{*}\left(z^{k}\right)=\left(-(m-k)^{2}\right) z^{n-m+k} .
$$

Then we adapt the previous computations:

$$
\begin{aligned}
\sum_{k \in \mathbb{Z}}\left(k^{2}\right)^{-s}\left(X Y^{*} z^{k}, z^{k}\right)_{L^{2}} & =\sum_{k \in \mathbb{Z}}\left(-(m-k)^{2}\right)\left(k^{2}\right)^{-s}\left(z^{n-m+k}, z^{k}\right)_{L^{2}} \\
& = \begin{cases}0 & \text { if } n \neq m, \\
-m^{2}-2 \sum_{k \in \mathbb{N} *} k^{-2 s+2}+m^{2} k^{-2 s} & \text { if } n=m .\end{cases}
\end{aligned}
$$

By the way, passing from the complex Fourier Basis to the real Fourier basis,

$$
\operatorname{tr}^{\Delta}\left(X Y^{*}\right)=-2 \zeta(-2)(u, v)_{L^{2}}+(-1-2 \zeta(0))(u, v)_{H_{0}^{1}}=0 .
$$

(3) Since $a$ and $X$ are even-even class, we thave that $\operatorname{tr}^{\Delta}(a X)=\operatorname{tr}^{\Delta}(X a)$. Setting $a=z^{n}$ and $X=u \frac{d}{d x}$, with $u=z^{m}$, we compute

$$
\begin{aligned}
\sum_{k \in \mathbb{Z}} i k\left(k^{2}\right)^{-s}\left(z^{n} z^{m} z^{k}, z^{k}\right)_{L^{2}} & =\sum_{k \in \mathbb{Z}} i k . k^{-2 s}\left(z^{n+m+k}, z^{k}\right)_{L^{2}} \\
& = \begin{cases}0 & \text { if } n \neq-m, \\
\sum_{k \in \mathbb{Z}} i k . k^{-2 s}=0 & \text { if } n=-m .\end{cases}
\end{aligned}
$$

By the way,

$$
\operatorname{tr}^{\Delta}(a X)=\operatorname{tr}^{\Delta}(X a)=0
$$




\subsection{Extension of the Hilbart-Schmidt metric to $F C l$.}

Theorem 4.4. The Hilbert-Schmidt definite positive Hermitian product

$$
(A, B)_{H S}=\operatorname{tr}\left(A B^{*}\right)
$$

which is positive, definite metric on $C^{-1}\left(S^{1}, V\right)$ extends:

- to a Hermitian, non degenerate form on $C l\left(S^{1}, V\right)$ by

$$
(A, B) \mapsto(A, B)_{\Delta}=\operatorname{tr}^{\Delta}\left(A B^{*}\right) ;
$$

- to a Hermitian, non degenerate form on $C l_{e e}\left(S^{1}, V\right)$ by

$$
(A, B) \mapsto(A, B)_{\Delta}=\operatorname{tr}^{\Delta}\left(A B^{*}\right)
$$

- to a $(\mathbb{R}-)$ bilinear, symmetric non degenerate form on

$$
C l\left(S^{1}, V\right) \oplus \operatorname{Vect}\left(S^{1}\right)
$$

by

$$
(A, B) \mapsto \mathfrak{R e}(A, B)_{\Delta}=\mathfrak{R e}\left(\operatorname{tr}^{\Delta}\left(A B^{*}\right)\right),
$$

where $A=a+u, B=b+v$, with $(a, b) \in C l\left(S^{1}, V\right)$ and $(u, v) \in \operatorname{Vect}\left(S^{1}\right)$.

Proof. We proceed set by set, following the order of the statement of the Theorem.

On $C l\left(S^{1}, V\right)$ : The formula $\operatorname{tr}\left(A B^{*}\right)$ gives obviously a sesquilinear form. We prove first that the form is Hermitian: Let $(A, B) \in C l\left(S^{1}, V\right)$.

$$
\operatorname{tr}^{\Delta}\left(B A^{*}\right)=\operatorname{tr}^{\Delta}\left(\left(A B^{*}\right)^{*}\right)=\overline{\operatorname{tr}^{\Delta}\left(A B^{*}\right)} .
$$

Let us then prove that it is non-degenerate. Let

$$
A \in C l\left(S^{1}, V\right), \quad u \in C^{\infty}\left(S^{1}, V\right) \cap(\operatorname{Im} A-\{0\})
$$

which is the image of a function $x$ such that $\|x\|_{L^{2}}=1$, and let $p_{x}$ be the $L^{2}$-orthogonal projection on the $\mathbb{C}$-vector space spanned by $x$. Then, let $\left(e_{k}\right)_{k \in \mathbb{N}}$ be an orthonormal base with $e_{0}=x$. For $\mathfrak{R e}(s) \geqslant 2 \operatorname{ord}(A)+2$, we observe, applying commutation relations of the usual trace of trace-class operators, the following:

$$
\begin{aligned}
\operatorname{tr}\left(A\left(p_{x} A\right)^{*} \Delta^{-s}\right) & =\operatorname{tr}\left(\Delta^{-s / 2} A\left(A p_{x}\right)^{*} \Delta^{-s / 2}\right) \\
& =\operatorname{tr}\left(\left(A p_{x}\right)^{*} \Delta^{-s / 2} \cdot \Delta^{-s / 2} A\right)=\operatorname{tr}\left(\left(A p_{x}\right)^{*} \Delta^{-s} A\right) .
\end{aligned}
$$

By the way, the meromorphic continuation to $\mathbb{C}$ of

$$
s \mapsto \operatorname{tr}\left(\left(A p_{x}\right)^{*} \Delta^{-s} A\right)
$$

exist and coincide with the meromorphic continuation of

$$
s \mapsto \operatorname{tr}\left(A\left(A p_{x}\right)^{*} \Delta^{-s}\right),
$$

in particular at $s=0$. 


$$
\begin{aligned}
\operatorname{tr}\left(\left(p_{x} A\right)^{*} \Delta^{-s} A\right) & =\sum_{k \in \mathbb{N}}\left(\left(p_{x} A\right)^{*} \Delta^{-s} A e_{k}, e_{k}\right)_{L^{2}}=\sum_{k \in \mathbb{N}}\left(\Delta^{-s} A e_{k}, A p_{x} e_{k}\right)_{L^{2}} \\
& =\left(\Delta^{-s} A x, A x\right)_{L^{2}}=\left(\Delta^{-s / 2} u, \Delta^{-s / 2} u\right)_{L^{2}} .
\end{aligned}
$$

By the way, since $\lim _{s \rightarrow 0} \Delta^{-s / 2}=\operatorname{Id}$ for weak convergence,

$$
\begin{aligned}
\operatorname{tr}^{\Delta}\left(A\left(A p_{x}\right)\right) & =\lim _{s \rightarrow 0} \operatorname{tr}\left(A\left(A p_{x}\right)^{*} \Delta^{-s}\right) \\
& =\lim _{s \rightarrow 0}\left(\Delta^{-s / 2} u, \Delta^{-s / 2} u\right)_{L^{2}}=\|u\|_{L^{2}}^{2} \neq 0 .
\end{aligned}
$$

The operator $A p_{x}$ is a smoothing (rank 1) operator, and hence is in $C l\left(S^{1}, V\right)$, which ends the proof.

On $C l_{e e}\left(S^{1}, V\right)$ and on $C l\left(S^{1}, V\right) \oplus \operatorname{Vect}\left(S^{1}\right)$ : The same arguments as before hold for non-degeneracy on both

$$
C l_{e e}\left(S^{1}, V\right) \text { and } \quad C l\left(S^{1}, V\right) \oplus \operatorname{Vect}\left(S^{1}\right) .
$$

The rest of the arguments follow from the inclusions

$$
C l_{e e}\left(S^{1}, V\right) \subset C l\left(S^{1}, V\right) \text { and } C l\left(S^{1}, V\right)+\operatorname{Vect}\left(S^{1}\right)=C l\left(S^{1}, V\right) .
$$

Remark 4.5. We remark that $(\cdot, \cdot)_{\Delta}$ is bilinear, non degenerate but not positive. Indeed, from relation (1) of Lemma $4.2, C^{\infty}\left(S^{1}, M_{n}(\mathbb{C})\right)$ is an isotropic Lie subalgebra for $(\cdot, \cdot)_{\Delta}$ which proves that this $\mathbb{R}$-bilinear symmetric form is not positive.

From the Lie algebra $C l\left(S^{1}, V\right) \oplus \operatorname{Vect}\left(S^{1}\right)$, we then span by rightinvariant action of $F C l^{*}\left(S^{1}, V\right)$ on $T F C l^{*}\left(S^{1}, V\right)$ a right-invariant pseudometric. For this goal, the Lie algebra elements are identified as infinitesimal paths, and actions and Lie brackets are those derived from the coadjoint action (and right-Lie bracket) of $F C l^{*}\left(S^{1}, V\right)$ on $C l\left(S^{1}, V\right) \oplus \operatorname{Vect}\left(S^{1}\right)$, while we consider the trivial mapping defined by the sum

$$
C l\left(S^{1}, V\right) \oplus \operatorname{Vect}\left(S^{1}\right) \rightarrow C l\left(S^{1}, V\right)=C l\left(S^{1}, V\right)+\operatorname{Vect}\left(S^{1}\right)
$$

in order to compute $\mathfrak{R e}(\cdot, \cdot)_{\Delta}$. The same constructions hold for the pseudoHermitian metric $(\cdot, \cdot)_{\Delta}$ on $C l^{*}\left(S^{1}, V\right)$.

Definition 4.6. Let $A \in F C l^{0, *}\left(S^{1}, V\right)$ and $a \in C l^{0}\left(S^{1}, V\right) \oplus \operatorname{Vect}\left(S^{1}\right)$. We denote by $R_{A}(a)$ the (right-)action by composition

$$
R_{A}(a)=a \circ A .
$$

Then, identifying $T_{A} F C l^{0, *}\left(S^{1}, V\right)$ with $R_{A}\left(C l^{0}\left(S^{1}, V\right) \oplus \operatorname{Vect}\left(S^{1}\right)\right)$ we set a smooth pseudo-Riemannain metric on $T F C l^{0, *}\left(S^{1}, V\right)$ by defining for

$$
(a, b) \in\left(C l^{0}\left(S^{1}, V\right) \oplus \operatorname{Vect}\left(S^{1}\right)\right)^{2},
$$


and hence for $\left(R_{A}(a), R_{A}(b)\right) \in\left(T_{A} F C l^{0, *}\left(S^{1}, V\right)\right)^{2}$,

$$
\left(R_{A}(a), R_{A}(b)\right)_{\Delta, A}=(a, b)_{\Delta} .
$$

5. In SEARCH OF PSEUdo-Hermitian CONNECTIONS FOR $(\cdot, \cdot)_{\Delta}$

There exists some difficulties in describing the whole space of connection 1-forms $\Omega^{1}\left(F C l_{\operatorname{Diff}\left(S^{1}\right)}\left(S^{1}, V\right), C l\left(S^{1}, V\right) \rtimes \operatorname{Vect}\left(S^{1}\right)\right)$. Indeed the space of smooth linear maps acting on $C l\left(S^{1}, V\right)$ is actually not well-understood to our knowledge. In particular, finding an adjoint of the adjoint map for $(\cdot, \cdot)_{\Delta}$ fails apparently due to the non-traciality of $\operatorname{tr}^{\Delta}$. We consider here a class of connections where this smooth linear endomorphism is defined by composition by a smoothing operator. The resulting technical simplifications enables us to get pseudo-Hermitian connections for $(\cdot, \cdot)_{\Delta}$. Most of them can be easily adapted to get pseudo-Riemannian connections for $\mathfrak{R e}(\cdot, \cdot)_{\Delta}$.

5.1. A class of connections. Let us define now, for $w \in C l\left(S^{1}, V\right)$ such that $\forall(a, b) \in C l\left(S^{1}, V\right)$,

$$
\Theta_{a}^{w} b=b[a, w]
$$

Proposition 5.2. The curvature of $\Theta^{w}$ reads as

$$
\Omega_{\Theta^{w}}(a, b) c=[w b w, a]-[w a w, b]+[w a, w b]-[w b, a w]-[[b, a] w]
$$

for $(a, b) \in \mathcal{F} C l\left(S^{1}, V\right)$.

Proof. Let $(a, b, c) \in C l\left(S^{1}, V\right)$.

$$
\begin{aligned}
\Omega_{\Theta^{w}}(a, b) c & =\left[\Theta_{a}^{w}, \Theta_{b}^{w}\right] c-\Theta_{[a, b]}^{w} c=c[b, w][a, w]-c[a, w][b, w]-c[[b, a], w] \\
& =c[w b w, a]-c[w a w, b]+c[w a, w b]-c[w b, a w]-c[[b, a] w] .
\end{aligned}
$$

Let us analyze the connection $\Theta^{w}$ with $w=i \epsilon(D)$.

Theorem 5.3. $\Theta^{i \epsilon(D)}$ is a $C l^{-\infty}\left(S^{1}, V\right)$-valued connection.

Proof. It follows directly from the fact that $[a, i \epsilon(D)] \in C l^{-\infty}\left(S^{1}, V\right)$.

\subsection{Pseudo-Hermitian connections associated with a skew-adjoint} pseudodifferential operator. Let $w \in C l\left(S^{1}, V\right)$ such that $w^{*}=-w$. For example, one can consider the example $w=i \epsilon(D)$.

Lemma 5.5. For all $a, w \in C l\left(S^{1}, V\right)$ such that $w^{*}=-w, \Theta_{a^{*}}^{w}$ is the adjoint of $\Theta_{a}^{s}$ for $(\cdot, \cdot)_{\Delta}$. 
Proof. Let $(a, b, c) \in C l^{-\infty}\left(S^{1}, V\right)^{3}$. Then

$$
\begin{aligned}
\left.\left(\Theta_{a}^{w} b, c\right)\right)_{\Delta} & =\operatorname{tr}^{\Delta}\left(b[a, w] c^{*}\right)=\operatorname{tr}^{\Delta}\left(b\left(\left[a^{*}, w\right]\right)^{*} c^{*}\right) \\
& =\operatorname{tr}^{\Delta}\left(b\left(c\left[a^{*}, w\right]\right)^{*}\right)=\operatorname{tr}^{\Delta}\left(b\left(\Theta_{a^{*}} c\right)^{*}\right) .
\end{aligned}
$$

Let us now analyze

$$
(a, b) \in C l\left(S^{1}, \mathbb{C}\right)^{2} \mapsto \theta_{a}^{w} b=b\left[a-a^{*}, w\right]=\left(\Theta_{a}^{w}-\Theta_{a^{*}}^{w}\right)(b) .
$$

Theorem 5.6. $\theta^{w}$ is the connection 1-form of a pseudo-Hermitian connection of $(\cdot, \cdot)_{\Delta}$.

Proof. Let $(a, b, c) \in C l\left(S^{1}, \mathbb{C}\right)^{3}$. Then

$$
\begin{aligned}
\left(\theta_{a}^{w} b, c\right)_{\Delta} & =\left(\left(\Theta_{a}^{w}-\Theta_{a^{*}}^{w}\right) b, c\right)_{\Delta}=\left(\left(\Theta_{a}^{w}, c\right)_{\Delta}-\left(\left(\Theta_{a^{*}}^{w}\right) b, c\right)_{\Delta}\right. \\
& =\left(\left(b, \Theta_{a^{*}}^{w} c\right)_{\Delta}-\left(b, \Theta_{a}^{w} c\right)_{\Delta}=-\left(b, \theta_{a^{*}}^{w} c\right)_{\Delta} .\right.
\end{aligned}
$$

Hence

$$
\left(\theta_{a}^{w} b, c\right)_{\Delta}+\left(b, \theta_{a^{*}}^{w} c\right)_{\Delta}=0 .
$$

Remark 5.7. $\theta^{i \epsilon(D)}$ is $C l^{-\infty}\left(S^{1}, V\right)$-valued as $\Theta^{i \epsilon(D)}$ is.

5.8. On another class $C l^{-\infty}\left(S^{1}, V\right)$-connections. Motivated by the previous example of $C l^{-\infty}\left(S^{1}, V\right)$, let us now give families of $C l^{-\infty}\left(S^{1}, V\right)$ connections which a priori do not include the connections $\theta^{i \epsilon(D)}$ and $\Theta^{i \epsilon(D)}$. Let us define now, for $s \in C l^{-\infty}$ and for all $(a, b) \in C l\left(S^{1}, V\right)$,

$$
\Theta_{a}^{s, l} b=s a s^{*} b, \quad \Theta_{a}^{s, r} b=b s a s^{*}, \quad \Theta_{a}^{s,[]} b=\left[\text { sas }^{*}, b\right] .
$$

Let us describe here their associated class of pseudo-Riemannian connections for $(\cdot, \cdot)_{\Delta}$ along the lines of the previous section. Let $s \in C l^{-\infty}\left(S^{1}, V\right)$ be a smoothing operator. Let $a, b \in C l\left(S^{1}, V\right)^{2}$ and let

$$
\begin{aligned}
\theta_{a}^{s,[]} b & =\Theta_{a}^{s,[]} b-\Theta_{a^{*}}^{s,[]} b=\left[s\left(a-a^{*}\right) s^{*}, b\right], \\
\theta_{a}^{s, l} & =\Theta_{a}^{s, l} b-\Theta_{a^{*}}^{s, l} b=s\left(a-a^{*}\right) s^{*} b, \\
\theta_{a}^{s, r} & =\Theta_{a}^{s, r} b-\Theta_{a^{*}}^{s, r} b=b s\left(a-a^{*}\right) s^{*} .
\end{aligned}
$$

Lemma 5.9. For all $a \in C l\left(S^{1}, V\right)$ and $s \in C l^{-\infty}\left(S^{1}, V\right)$ we have that

- $\Theta_{a^{*}}^{s, l}$ is the adjoint of $\Theta_{a}^{s, l}$ for $(\cdot, \cdot)_{\Delta}$,

- $\Theta_{a^{*}}^{s, r}$ is the adjoint of $\Theta_{a}^{s, l}$ for $(\cdot, \cdot)_{\Delta}$,

- $\Theta_{a^{*}}^{s,[]}$ is the adjoint of $\Theta_{a}^{s, l}$ for $(\cdot, \cdot)_{\Delta}$.

Proof. Let $(a, b, c) \in C l\left(S^{1}, \mathbb{C}\right)^{3}$. Then

$$
\begin{aligned}
\left(\Theta_{a}^{s, l} b, c\right)_{\Delta} & =\left(\operatorname{sas}^{*} b, c\right)_{\Delta}=\operatorname{tr}^{\Delta}\left(\operatorname{sas}^{*} b c^{*}\right) \\
& =\operatorname{tr}^{\Delta}\left(b c^{*} \operatorname{sas}^{*}\right) \text { because } \operatorname{sas}^{*} \in C l^{-\infty}\left(S^{1}, V\right)
\end{aligned}
$$




$$
=\operatorname{tr}^{\Delta}\left(b\left(s a^{*} s^{*} c\right)^{*}\right),
$$

which proves the first point. Furthermore,

$$
\left(\Theta_{a}^{s, r} b, c\right)_{\Delta}=\left(b_{s a s}^{*}, c\right)_{\Delta}=\operatorname{tr}^{\Delta}\left(\text { bsas }^{*} c^{*}\right)=\operatorname{tr}^{\Delta}\left(b\left(c s a^{*} s^{*}\right)^{*}\right) .
$$

which proves the second point. The third pont is proved straightway by the remark $\Theta_{a}^{s,[]}=\Theta_{a}^{s, l}-\Theta_{a}^{s, r}$

Theorem 5.10. Then $\theta^{s,[]}, \theta^{s, l}$ and $\theta^{s, r}$, define three right-invariant pseudoHermitian connections on $\mathrm{FCl}\left(S^{1}, V\right)$.

Proof. This follows from Lemma 5.9.

\section{LAST REMARKS}

6.1. $\operatorname{Diff}\left(S^{1}\right)$ versus $\operatorname{Diff}_{+}\left(S^{1}\right)$. The group of diffeomorphims $\operatorname{Diff}\left(S^{1}\right)$ splits into two connected components

$$
\operatorname{Diff}\left(S^{1}\right)=\operatorname{Diff}_{+}\left(S^{1}\right) \bigsqcup \operatorname{Diff}_{-}\left(S^{1}\right)
$$

where Diff_ $\left(S^{1}\right)$ is the space of diffeomorphisms which reverse the orientation of $S^{1}$. Among these diffeomorphisms, there is the conjugate map

$$
z \in S^{1} \mapsto \bar{z}
$$

which induces an involution

$$
\text { Conj : }(z \mapsto f(z)) \in L^{2}\left(S^{1}, V\right) \mapsto(z \mapsto f(\bar{z})) \in L^{2}\left(S^{1}, V\right)
$$

that decomposes blockwise in $L^{2}\left(S^{1}, V\right)=E_{-} \oplus E_{0} \oplus E_{+}$as

$$
\text { Conj }=\left(\begin{array}{ccc}
0 & 0 & * \\
0 & \operatorname{Id}_{E_{0}} & 0 \\
* & 0 & 0
\end{array}\right)
$$

and in $H_{+} \oplus H_{-}$, for any choice made for $H_{+}$and $H_{-}$(see Section 3.1) as

$$
\text { Conj }=\left(\begin{array}{ll}
\text { Conj }_{++} & \text {Conj }_{+-} \\
\text {Conj }_{-+} & \text {Conj }_{--}
\end{array}\right)
$$

where Conj $j_{++}$and Conj_- are finite rank, smoothing operators. We have that

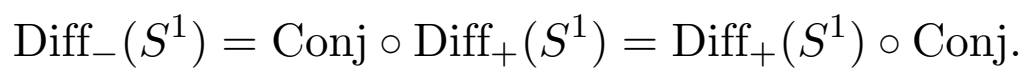

By the way, given $A \in F C l_{\operatorname{Diff}\left(S^{1}\right)}\left(S^{1}, V\right)$, if the phase diffeomorphism $g$ of $A$ is orientation preserving, then, under the blockwise decomposition $H_{+} \oplus H_{-}$,

$$
A=\left(\begin{array}{cc}
A_{++} & A_{+-} \\
A_{-+} & A_{--}
\end{array}\right),
$$


$A_{+-}$and $A_{-+}$are smoothing operators due to [23], and if $g \in \operatorname{Diff}_{-}\left(S^{1}\right)$, $A_{++}$and $A_{--}$are smoothing operators.

6.2. The Schwinger cocycle and the connection $\Theta^{i \epsilon}$. Let us make the two following remarks

Proposition 6.3. Let $(a, b) \in C l\left(S^{1}, V\right) \rtimes \operatorname{Vect}\left(S^{1}\right)$. Then

$$
c_{s}(a, b)=-i \operatorname{tr}^{\Delta}\left(\Theta_{a}^{i \epsilon} b\right)=\operatorname{tr}^{\Delta}\left(\Theta_{a}^{i \epsilon} \Theta_{b}^{i \epsilon} \epsilon(D)\right) .
$$

Proof. We compute independently

$$
\operatorname{tr}^{\Delta}\left(\Theta_{a}^{i \epsilon} b\right)=\operatorname{tr}^{\Delta}(b[a, i \epsilon(D)])=i \operatorname{tr}^{\Delta}([a, \epsilon(D)] b)
$$

since $[a, \epsilon(D)] \in C l^{-\infty}\left(S^{1}, V\right)$, and

$$
\begin{aligned}
\operatorname{tr}^{\Delta}\left(\Theta_{a}^{i \epsilon} \Theta_{b}^{i \epsilon} \epsilon(D)\right) & =\operatorname{tr}^{\Delta}(\epsilon(D)[b, i \epsilon(D)][a, i \epsilon(D)]) \\
& =-c_{s}(b, a)=c_{s}(a, b) .
\end{aligned}
$$

Remark 6.4. When defining a smoothing connection $\theta$ on

$$
C l\left(S^{1}, V\right) \rtimes \operatorname{Vect}\left(S^{1}\right),
$$

we define a map with values on the first component of the product

$$
C l\left(S^{1}, V\right) \times \operatorname{Vect}\left(S^{1}\right) .
$$

Theorem 6.5. The Schwinger cocycle $c_{s}$ has the same cohomology class as

$$
c_{1}^{i \epsilon}:(a, b) \in C l\left(S^{1}, V\right)^{2} \mapsto \frac{1}{2} \operatorname{tr}^{\Delta}\left(\Omega^{i \epsilon}(a, b) \epsilon(D)\right)
$$

where $\Omega^{i \epsilon}$ is the curvature of $\Theta^{i \epsilon}$.

Proof. We have, $\forall(a, b) \in C l\left(S^{1}, V\right)^{2}$ :

$$
\begin{aligned}
\operatorname{tr}^{\Delta}\left(\Omega^{i \epsilon}(a, b) \epsilon(D)\right)= & -\operatorname{tr}^{\Delta}(\epsilon(D)[b, \epsilon(D)][a, \epsilon(D)]- \\
& -\epsilon(D)[a, \epsilon(D)][b, \epsilon(D)]-\epsilon(D)[[b, a], \epsilon(D)]) \\
= & -\operatorname{tr}^{\Delta}(\epsilon(D)[b, \epsilon(D)][a, \epsilon(D)])+ \\
& +\operatorname{tr}^{\Delta}(\epsilon(D)[a, \epsilon(D)][b, \epsilon(D)])-\operatorname{tr}^{\Delta}(\epsilon(D)[[b, a], \epsilon(D)]) \\
= & c_{s}(a, b)+c_{s}(a, b)+(\delta \gamma)(a, b),
\end{aligned}
$$

where $\delta$ is the coboundary operator and

$$
\gamma: a \in C l\left(S^{1}, V\right) \mapsto \operatorname{tr}^{\Delta}(\epsilon(D)[a, \epsilon(D)])=c_{s}(a, \epsilon(D)) .
$$


6.6. On even-even $\operatorname{Diff}\left(S^{1}\right)$-pseudo-differential operators. Considering now

$$
F C l_{e e, \operatorname{Diff}\left(S^{1}\right)}\left(S^{1}, V\right)=C l_{e e}^{*}\left(S^{1}, V\right) \rtimes \operatorname{Diff}\left(S^{1}\right),
$$

we remark that the renormalized trace $\operatorname{tr}^{\Delta}$ is tracial on its Lie algebra $C l_{e e}\left(S^{1}, V\right) \rtimes \operatorname{Vect}\left(S^{1}\right)$, i.e.

$$
\forall(a, b) \in C l_{e e}\left(S^{1}, V\right), \quad \operatorname{tr}^{\Delta}([a, b])=0
$$

(representing $C l_{e e}\left(S^{1}, V\right) \rtimes \operatorname{Vect}\left(S^{1}\right)$ in $C l_{e e}\left(S^{1}, V\right)$ as in the rest of the text). This enables to state the following property:

Proposition 6.7. For all $a \in C l_{e e}\left(S^{1}, V\right)$ the adjoint map

$$
a d_{a}: b \mapsto a d_{a} b=[b, a]
$$

has an adjoint map for $(\cdot, \cdot)_{\Delta}$ given by

$$
a d_{a}^{*}=a d_{a^{*}}
$$

Proof. Let $(a, b, c) \in C l_{e e}\left(S^{1}, V\right)^{3}$. Then

$$
\begin{aligned}
\operatorname{tr}^{\Delta}\left(\left(a d_{a} b\right) c^{*}\right) & =\operatorname{tr}^{\Delta}\left([b, a] c^{*}\right)=\operatorname{tr}^{\Delta}\left(b a c^{*}\right)-\operatorname{tr}^{\Delta}\left(a b c^{*}\right) \\
& =\operatorname{tr}^{\Delta}\left(b a c^{*}\right)-\operatorname{tr}^{\Delta}\left(b c^{*} a\right)=\operatorname{tr}^{\Delta}\left(b\left(c a^{*}\right)^{*}\right)-\operatorname{tr}^{\Delta}\left(b\left(a^{*} c\right)^{*}\right) \\
& =\operatorname{tr}^{\Delta}\left(b\left(\left[c, a^{*}\right]\right)^{*}\right) .
\end{aligned}
$$

As a consequence, applying the arguments of [7] and especially those leading to [7, Proposition 1.7] to right-invariant vector fields on

$$
F C l_{e e, \operatorname{Diff}\left(S^{1}\right)}\left(S^{1}, V\right),
$$

we get:

Theorem 6.8. The pseudo-Riemannian metric $\mathfrak{R e}(\cdot, \cdot)_{\Delta}$ admits a unique pseudo-Riemannian, torsion-free (i.e. Levi-Civita) connection $\nabla^{\Delta}$ that reads as

$$
\nabla_{a}^{\Delta} b=\frac{1}{2}\left(a d_{a} b-a d_{a *} b-a d_{b^{*}} a\right)
$$

6.9. On bounded even-even $\operatorname{Diff}\left(S^{1}\right)$-pseudo-differential operators. We finish our remarks with the group of $\left(L^{2}\right.$-) bounded even-even $\operatorname{Diff}\left(S^{1}\right)$ pseudo-differential operators. Its Lie algebra

$$
C l_{e e}^{0}\left(S^{1}, V\right) \rtimes \operatorname{Vect}\left(S^{1}\right)
$$

also reads as

$$
C l_{e e}^{-1}\left(S^{1}, V\right) \oplus D O^{0}\left(S^{1}, V\right) \oplus\left(\operatorname{Vect}\left(S^{1}\right) \otimes \operatorname{Id}_{V}\right) .
$$


and the pseudo-Riemannian product $\mathfrak{R e}(\cdot, \cdot)_{\Delta}$ decomposes blockwise as

$$
\left(\begin{array}{ccc}
\mathfrak{R e}(\cdot, \cdot)_{H S} & * & * \\
* & 0 & 0 \\
* & 0 & 0
\end{array}\right),
$$

where $\mathfrak{R e}(\cdot, \cdot)_{H S}=\mathfrak{R e}\left((\cdot, \cdot)_{H S}\right)$ is the scalar product derived from the Hilbert-Schmidt Hermitian product $(\cdot, \cdot)_{H S}$.

\section{REFERENCES}

[1] Malcolm Adams, Tudor Ratiu, Rudolf Schmid. A Lie group structure for Fourier integral operators. Math. Ann., 276(1):19-41, 1986, doi: 10.1007/BF01450921.

[2] Malcolm Adams, Tudor Ratiu, Rudolf Schmid. A Lie group structure for pseudodifferential operators. Math. Ann., 273(4):529-551, 1986, doi: 10.1007/BF01472130.

[3] M. Adler. On a trace functional for formal pseudo differential operators and the symplectic structure of the Korteweg-de Vries type equations. Invent. Math., 50(3):219-248, 1978/79, doi: 10.1007/BF01410079.

[4] Juliane Bokobza-Haggiag. Opérateurs pseudo-différentiels sur une variété différentiable. Ann. Inst. Fourier (Grenoble), 19(1):125-177, x, 1969, http://www.numdam. org/item?id=AIF_1969__19_1_125_0.

[5] A. Cardona, C. Ducourtioux, J. P. Magnot, S. Paycha. Weighted traces on algebras of pseudo-differential operators and geometry on loop groups. Infin. Dimens. Anal. Quantum Probab. Relat. Top., 5(4):503-540, 2002, doi: 10.1142/S021902570200095X.

[6] James Eells, Jr. A setting for global analysis. Bull. Amer. Math. Soc., 72:751-807, 1966, doi: 10.1090/S0002-9904-1966-11558-6.

[7] Daniel S. Freed. The geometry of loop groups. J. Differential Geom., 28(2):223-276, 1988, http://projecteuclid.org/euclid.jdg/1214442279.

[8] Peter B. Gilkey. Invariance theory, the heat equation, and the Atiyah-Singer index theorem, volume 11 of Mathematics Lecture Series. Publish or Perish, Inc., Wilmington, DE, 1984.

[9] L. Hörmander. Fourier integral operators. i. Acta Mathematica, 127:79-189, 1971.

[10] Patrick Iglesias-Zemmour. Diffeology, volume 185 of Mathematical Surveys and Monographs. American Mathematical Society, Providence, RI, 2013, doi: 10.1090/surv/185.

[11] Christian Kassel. Le résidu non commutatif. In Séminaire Bourbaki : volume 1988/89, exposés 700-714, number 177-178 in Astérisque. Société mathématique de France, 1989, http://www.numdam.org/item/SB_1988-1989__31__199_0/. talk:708 (in fr).

[12] B. Khesin, R. Wendt. The Geometry of Infinite-Dimensional Groups. Springer Verlag, 2009, doi: 10.1007/978-3-540-77263-7.

[13] M. Kontsevich, S. Vishik. Determinants of elliptic pseudo-differential operators. 1994.

[14] M. Kontsevich, S. Vishik. Geometry of determinants of elliptic operators. Progr. Math., 131:173-197, 1995, doi: 10.1007/978-1-4612-2582-9_6.

[15] O.S. Kravchenko, B.A. Khesin. A central extension of the algebra of pseudo-differential symbols. Funct. Anal. Appl., 25(2):152-154, 1991, doi: 10.1007/bf01079603.

[16] Martin Laubinger. A Lie algebra for Frölicher groups. Indag. Math. (N.S.), 21(3-4):156174, 2011, doi: 10.1016/j.indag.2011.04.001.

[17] Matthias Lesch. On the noncommutative residue for pseudodifferential operators with log-polyhomogeneous symbols. Ann. Global Anal. Geom., 17(2):151-187, 1999, doi: 10.1023/A:1006504318696. 
[18] J-P. Magnot. The Kähler form on the loop group and the Radul cocycle on pseudodifferential operators. In Proceedings of the 24th international colloquium on group theorical methods in physics, Paris, France, 15-20 July 2002, volume 173, pages 671675. 2003.

[19] J-P. Magnot. Chern forms on mapping spaces. Acta Appl. Math., 91(1):67-95, 2006, doi: 10.1007/s10440-006-9016-7.

[20] J-P. Magnot. Difféologie du fibré d'holonomie d'une connexion en dimension infinie. $C$. R. Math. Acad. Sci. Soc. R. Can., 28(4):121-128, 2006.

[21] J-P. Magnot. Renormalized traces and cocycles on the algebra of $S^{1}$-pseudo-differential operators. Lett. Math. Phys., 75(2):111-127, 2006, doi: 10.1007/s11005-005-0033-7.

[22] J-P. Magnot. The Schwinger cocycle on algebras with unbounded operators. Bull. Sci. Math., 132(2):112-127, 2008, doi: 10.1016/j.bulsci.2006.07.006.

[23] J-P. Magnot. On $\operatorname{diff}(m)$-pseudodifferential operators and the geometry of non linear grassmannians. Mathematics, 4(1), 2016, doi: 10.3390/math4010001.

[24] J-P. Magnot. The group of diffeomorphisms of a non-compact manifold is not regular. Demonstr. Math., 51(1):8-16, 2018, doi: 10.1515/dema-2018-0001.

[25] J-P Magnot, E. G. Reyes. On the Cauchy problem for a Kadomtsev-Petviashvili hierarchy on non-formal operators and its relation with a group of diffeomorphisms. 2018.

[26] J-P. Magnot, E.G. Reyes. In N. Euler, M.C. Nucci, editors, Nonlinear systems and their remarkable mathematical structures, volume 2, chapter The Cauchy problem of the Kadomtsev-Petviashvili hierarchy and infinite-dimensional groups. CRC press, 2020, doi: 10.1201/9780429263743.

[27] Jean-Pierre Magnot, Enrique G. Reyes. Well-posedness of the Kadomtsev-Petviashvili hierarchy, Mulase factorization, and Frölicher Lie groups. Ann. Henri Poincaré, 21(6):1893-1945, 2020, doi: 10.1007/s00023-020-00896-3.

[28] J. Mickelsson. Wodzicki residue and anomalies on current algebras. In Integrable Models and Strings, volume 436, pages 123-135. 1994, doi: 10.1007/3-540-58453-6_7.

[29] Karl-Hermann Neeb. Towards a Lie theory of locally convex groups. Jpn. J. Math., 1(2):291-468, 2006, doi: 10.1007/s11537-006-0606-y.

[30] P. P. Ntumba, T. A. Batubenge. On the way to Frölicher Lie groups. Quaest. Math., 28(1):73-93, 2005, doi: 10.2989/16073600509486116.

[31] Hideki Omori. Infinite-dimensional Lie groups, volume 158 of Translations of Mathematical Monographs. American Mathematical Society, Providence, RI, 1997, doi: $10.1090 / \mathrm{mmono} / 158$. Translated from the 1979 Japanese original and revised by the author.

[32] Sylvie Paycha. Regularised integrals, sums and traces, volume 59 of University Lecture Series. American Mathematical Society, Providence, RI, 2012, doi: 10.1090/ulect/059. An analytic point of view.

[33] Sylvie Paycha. Paths towards an extension of Chern-Weil calculus to a class of infinite dimensional vector bundles. In Geometric and topological methods for quantum field theory, pages 81-143. Cambridge Univ. Press, Cambridge, 2013.

[34] Andrew Pressley, Graeme Segal. Loop groups. Oxford Mathematical Monographs. The Clarendon Press, Oxford University Press, New York, 1986. Oxford Science Publications.

[35] A. O. Radul. Lie algebras of differential operators, their central extensions and $W$ algebras. Funktsional. Anal. i Prilozhen., 25(1):33-49, 1991, doi: 10.1007/BF01090674.

[36] J. Schwinger. Field theory commutators. Phys. Rev. Lett., 3:296-297, Sep 1959, doi: 10.1103/PhysRevLett.3.296. 
[37] Simon Scott. Traces and determinants of pseudodifferential operators. Oxford Mathematical Monographs. Oxford University Press, Oxford, 2010, doi: 10.1093/acprof:oso/9780198568360.001.0001.

[38] R. T. Seeley. Complex powers of an elliptic operator. In Singular Integrals (Proc. Sympos. Pure Math., Chicago, Ill., 1966), pages 288-307. Amer. Math. Soc., Providence, R.I., 1967.

[39] Harold Widom. A complete symbolic calculus for pseudodifferential operators. Bull. Sci. Math. (2), 104(1):19-63, 1980.

[40] M. Wodzicki. Local invariants of spectral asymmetry. Invent. Math., 75(1):143-177, 1984, doi: 10.1007/BF01403095.

Received: September 11, 2020, accepted: October 28, 2020.

Jean-Pierre Magnot

LAREMA - UMR CNRS 6093, UNIVERSITÉ D'ANGERS,

2 Boulevard Lavoisier 49045 Angers Cedex 01 and Lycée Jeanne dArC, 30 avenue de Grande Bretagne, F-63000 Clermont-Ferrand

Email: jean-pierr.magnot@ac-clermont.fr

ORCID: orcid.org/0000-0002-3959-3443 